\title{
Supersolutions for parabolic equations with unbounded diffusion and its applications to some classes of parabolic and hyperbolic equations
}

\author{
Motohiro Sobajima* and Yuta Wakasugi ${ }^{\dagger}$
}

Abstract. This paper is concerned with supersolutions to parabolic equations of the form

$$
\partial_{t} U(x, t)-D(x) \Delta U(x, t)=0, \quad(x, t) \in \mathbb{R}^{N} \times(0, \infty),
$$

where $D \in C\left(\mathbb{R}^{N}\right)$ is positive. Under the behavior of the diffusion coefficient $D$ with polynomial order at spatial infinity, a family of supersolutions to $(0.1)$ with slowly decaying property at spatial infinity is provided. As a first application, weighted $L^{2}$ type decay estimates for the initial-boundary value problem of the parabolic equation

$$
\begin{cases}\partial_{t} v(x, t)-D(x) \Delta v(x, t)=0, & (x, t) \in \Omega \times(0, \infty), \\ v(x, t)=0, & (x, t) \in \partial \Omega \times(0, \infty), \\ v(x, 0)=v_{0}(x), & x \in \Omega\end{cases}
$$

are proved. The second application is the study of the exterior problem of wave equations with spacedependent damping terms of type

$$
\begin{cases}\partial_{t}^{2} u(x, t)-\Delta u(x, t)+a(x) \partial_{t} u(x)=0, & (x, t) \in \Omega \times(0, \infty), \\ u(x, t)=0, & (x, t) \in \partial \Omega \times(0, \infty), \\ u(x, 0)=u_{0}(x), \partial_{t} u(x, 0)=u_{1}(x), & x \in \Omega .\end{cases}
$$

By using supersolution provided above with $a(x)=D(x)^{-1}$, energy estimates for (0.3) with polynomial weight and diffusion phenomena are shown. There is a slight improvement comparing to the one in [24] about the assumption of the initial data.

Mathematics Subject Classification (2010): Primary: 35K20 Secondary: 35L20, 35B40.

Key words and phrases: parabolic equation with unbounded diffusion, supersolution, damped wave equation, spacedependent damping, diffusion phenomena.

\section{Introduction}

In this paper we consider positive supersolutions of the following parabolic equation

$$
\begin{cases}\partial_{t} U(x, t)-D(x) \Delta U(x, t)=0, & (x, t) \in \mathbb{R}^{N} \times(0, \infty), \\ U(x, 0)=w(x), & x \in \mathbb{R}^{N},\end{cases}
$$

where $N \in \mathbb{N}$ and the diffusion coefficient $D$ satisfies

$$
D \in C\left(\mathbb{R}^{N}\right), \quad D(x)>0, \quad \lim _{|x| \rightarrow \infty}\left(|x|^{-\alpha} D(x)\right)=D_{0}
$$

for some constants $\alpha \in \mathbb{R}$ and $D_{0}>0$. That is, we assume $D_{1}\langle x\rangle^{\alpha} \leq D(x) \leq D_{2}\langle x\rangle^{\alpha}$ for some $D_{1}, D_{2}>0$, where $\langle x\rangle=\sqrt{1+|x|^{2}}$. The initial value $w(x)$ also behaves polynomially at spatial infinity. We emphasize that the diffusion coefficient $D$ is allowed to be unbounded $(\alpha>0)$ and also decreasing $(\alpha<0)$.

${ }^{*}$ Department of Mathematics, Faculty of Science and Technology, Tokyo University of Science, 2641 Yamazaki, Noda-shi, Chiba, 278-8510, Japan, E-mail: msobajima1984@gmail.com

$\dagger$ Graduate School of Science and Engineering, Ehime University, 3, Bunkyo-cho, Matsuyama, Ehime, 790-8577, Japan, E-mail: wakasugi .yuta.vi@ehime-u.ac.jp. 
The notion of subsolutions and supersolutions for elliptic and parabolic problems are well-known. They are essentially provided from the maximum principle of the corresponding problems as typified by the positivity of the solutions. Nowadays, so-called supersolution-subsolution methods for such problems are understood as powerful tools to analyse the existence and uniqueness of solutions and to discover profiles of them. (For the detail, see e.g., Gilbarg-Trudinger [5] for elliptic problems and Quittner-Souplet [19] for parabolic problems, and their references therein).

Here we shall focus our attention to the notion of supersolutions for parabolic problems. For instance, in the case of the following problem

$$
\begin{cases}\partial_{t} u(x, t)-\Delta u(x, t)=(u(x, t))^{p} & (x, t) \in \Omega \times(0, T), \\ u(x, t)=0, & (x, t) \in \partial \Omega \times(0, T), \\ u(x, 0)=u_{0}(x) \geq 0, & x \in \Omega,\end{cases}
$$

the function $\bar{u} \in C^{2}(\Omega \times[0, T))$ is called supersolution of (1.3) if

$$
\begin{cases}\partial_{t} \bar{u}(x, t)-\Delta \bar{u}(x, t) \geq(\bar{u}(x, t))^{p} & (x, t) \in \Omega \times(0, T), \\ \bar{u}(x, t) \geq 0, & (x, t) \in \partial \Omega \times(0, T), \\ \bar{u}(x, 0) \geq u_{0}(x), & x \in \Omega .\end{cases}
$$

Once we find a supersolution of (1.3), then we can immediately obtain an estimate for solutions in a pointwise sense. By using this structure, Weissler [28] proved single-point blowup of solutions to (1.3). The structure of supersolutions also can be found in the study of nonlinear parabolic systems. We only quote Levine [12], Lu-Sleeman [13] and Ishige-Kawakami-Sierżęga [9].

The references stated above suggest that a criterion of construction of supersolutions (subsolutions) for general problem enables us to reach a further detailed analysis of profiles of solutions.

The purpose of the present paper is to give a family of supersolutions to the problem (1.1) and to discuss applications of them to weighted $L^{2}$ type decay estimates for initial-boundary value problems of parabolic equations and diffusion phenomena for the hyperbolic equations with space-dependent damping.

To state our main result, we would give the definition of supersolutions to (1.1) as follows.

Definition 1.1. For given initial value $w \in C(\Omega)$, a function $\bar{U}$ is said to be a supersolution of (1.1) if $\bar{U} \in C^{2}(\bar{\Omega} \times[0, \infty))$ satisfies

$$
\begin{cases}\partial_{t} \bar{U}(x, t)-D(x) \Delta \bar{U}(x, t) \geq 0, & (x, t) \in \Omega \times(0, \infty), \\ \bar{U}(x, t) \geq 0, & (x, t) \in \partial \Omega \times(0, \infty), \\ \bar{U}(x, 0) \geq w(x), & x \in \Omega .\end{cases}
$$

We are interested in supersolutions with polynomially decaying property at spatial infinity. Therefore, we do not handle supersolutions similar to Gaussian function $t^{-N / 2} \exp \left(-|x|^{2} / 4 t\right)$. The following assertion is the main result of this paper, which deals with the supersolutions of diffusion equation (1.1) with polynomially decaying property at spatial infinity.

Theorem 1.2. Assume that $D$ satisfies (1.2) for $\alpha \in(-\infty, \min \{2, N\})$. Then for $\sigma \in\left(0, \frac{N-\alpha}{2}\right)$, there exist a supersolution $\bar{U}_{D, \sigma} \in C^{2}(\bar{\Omega} \times[0, \infty))$ with $w(x)=\langle x\rangle^{-2 \sigma}$ and positive constants $c_{D, \sigma}, C_{D, \sigma}$ and $C_{D, \sigma}^{\prime}$ such that

$$
\begin{gathered}
c_{D, \sigma}\left(1+t+\langle x\rangle^{2-\alpha}\right)^{-\frac{2 \sigma}{2-\alpha}} \leq \bar{U}_{D, \sigma}(x, t) \leq C_{D, \sigma}\left(1+t+\langle x\rangle^{2-\alpha}\right)^{-\frac{2 \sigma}{2-\alpha}} \\
\left|\partial_{t} \bar{U}_{D, \sigma}(x, t)\right| \leq C_{D, \sigma}^{\prime}\left(1+t+\langle x\rangle^{2-\alpha}\right)^{-\frac{2 \sigma}{2-\alpha}-1}
\end{gathered}
$$


Remark 1.1. Here we point out about the case $\sigma=\frac{N-\alpha}{2}$. Formally, we consider the equation $\partial_{t} u=|x|^{\alpha} \Delta u$. This has the (super)solution

$$
(1+t)^{-\frac{N-\alpha}{2-\alpha}} \exp \left(-\frac{|x|^{2-\alpha}}{(2-\alpha)^{2}(1+t)}\right) .
$$

In contrast, if $\sigma<\frac{N-\alpha}{2}$, then the corresponding self-similar solution of $\partial_{t} u=|x|^{\alpha} \Delta u$ has the following form

$$
(1+t)^{-\frac{2 \sigma}{2-\alpha}} \exp \left(-\frac{|x|^{-\alpha}}{(2-\alpha)^{2}(1+t)}\right) M\left(\frac{N-\alpha-2 \sigma}{2-\alpha}, \frac{N-\alpha}{2-\alpha} ; \frac{|x|^{-\alpha}}{(2-\alpha)^{2}(1+t)}\right) \approx\left(1+t+\frac{|x|^{2-\alpha}}{(2-\alpha)^{2}}\right)^{-\frac{2 \sigma}{2-\alpha}},
$$

where $M(\cdot, \cdot ; \cdot)$ is the Kummer confluent hypergeometric function (see Definition 2.1, below, and also [24]). In view of the explicit representation of self-similar solution, the condition $\sigma \leq \frac{N-\alpha}{2}$ is required to ensure the positivity of self-similar solutions and the restriction $\sigma<\frac{N-\alpha}{2}$ is necessary for the polynomially decaying profile of self-similar solutions.

As a first application to supersolutions in Theorem 1.2, we provide a weighted $L^{2}$-type decay estimate for the initial-boundary value problem of (1.1), that is,

$$
\begin{cases}\partial_{t} v(x, t)-D(x) \Delta v(x, t)=0, & (x, t) \in \Omega \times(0, \infty) \\ v(x, t)=0, & (x, t) \in \partial \Omega \times(0, \infty) \\ v(x, 0)=v_{0}(x), & x \in \Omega\end{cases}
$$

under the assumption (1.2). Here we consider the problem (1.6) in an exterior domain in $\mathbb{R}^{N}$. In this case, the Friedrichs extension of the corresponding elliptic operator $D \Delta$ generates an analytic semigroup $\{T(t)\}_{t \geq 0}$ on a weighted $L^{2}$ space (see Section 4). We say that $v=T(t) v_{0}$ is the solution of (1.6). The properties of solutions to this problem, for instance $L^{p}-L^{q}$ type estimates, can be found in literature (see e.g., Ioku-Metafune-Sobajima-Spina [10], Sobajima-Wakasugi [21, 23]). In the present paper, we will give the following assertion.

Proposition 1.3. Assume that D satisfies (1.2) with $\alpha \in(-\infty, \min \{2, N\})$. Let $v_{0}$ satisfy $\langle x\rangle^{\sigma-\frac{\alpha}{2}} v_{0} \in L^{2}(\Omega)$ for some $\sigma \in\left(0, \frac{N-\alpha}{2}\right)$. Then the solution $v$ of the problem (1.6) satisfies

$$
\left\|\langle x\rangle^{-\frac{\alpha}{2}} v(t)\right\|_{L^{2}(\Omega)} \leq C(1+t)^{-\frac{\sigma}{2-\alpha}}\left\|\langle x\rangle^{\sigma-\frac{\alpha}{2}} v_{0}\right\|_{L^{2}(\Omega)}, \quad t \geq 0
$$

for some positive constant $C$ independent of $v_{0}$.

Remark 1.2. Proposition 1.3 comes from the following formal computation via integration by parts twice: for positive $\Phi \in C^{2}\left(\mathbb{R}^{N+1}\right)$,

$$
\begin{aligned}
\frac{d}{d t} \int_{\Omega} \frac{v^{2}}{\Phi D} d x & =2 \int_{\Omega} \frac{v \partial_{t} v}{\Phi D} d x-\int_{\Omega} \frac{v^{2} \partial_{t} \Phi}{\Phi^{2} D} d x \\
& =2 \int_{\Omega} \frac{v \Delta v}{\Phi} d x-\int_{\Omega} \frac{v^{2} \partial_{t} \Phi}{\Phi^{2} D} d x \\
& =-2 \int_{\Omega}\left|\nabla\left(\frac{v}{\Phi}\right)\right|^{2} \Phi d x-\int_{\Omega} \frac{v^{2}\left(\partial_{t} \Phi-D \Delta \Phi\right)}{\Phi^{2} D} d x
\end{aligned}
$$

From the above estimate, one can find a weighted $L^{2}$-estimate of $v$ through the $L^{\infty}$-estimate of supersolution $\Phi$. However, this is not clear because of the regularity of $v$ to verify the computation with integration by parts. Instead of the difficulty stated above, the desired estimate is proved via semigroup approach as an application of the Trotter-Kato approximation theorem. 
The second application is the analysis of wave equations with space-dependent damping term:

$$
\begin{cases}\partial_{t}^{2} u(x, t)-\Delta u(x, t)+a(x) \partial_{t} u(x)=0, & (x, t) \in \Omega \times(0, \infty), \\ u(x, t)=0, & (x, t) \in \partial \Omega \times(0, \infty), \\ u(x, 0)=u_{0}(x), \partial_{t} u(x, 0)=u_{1}(x), & x \in \Omega,\end{cases}
$$

where $a(x)=D(x)^{-1}$, that is, $a \in C(\bar{\Omega})$ satisfying

$$
a(x)>0, \quad \lim _{|x| \rightarrow \infty}\left(|x|^{\alpha} a(x)\right)=a_{0}\left(=D_{0}^{-1}\right) .
$$

If $a \equiv 1$, then (1.7) is the usual damped wave equation. This is motivated by the derivation by Cattaneo [2] and Vernotte [26] as the approximation of heat equation with finite propagation property. Actually, it is known that if $u_{0}$ and $u_{1}$ are compactly supported smooth function, then so-called diffusion phenomena occurs, that is, the solution of (1.7) for $t \gg 1$ can be approximated by the solution of

$$
\begin{cases}a(x) \partial_{t} v(x)-\Delta v(x, t)=0, & (x, t) \in \Omega \times(0, \infty), \\ v(x, t)=0, & (x, t) \in \partial \Omega \times(0, \infty), \\ v(x, 0)=u_{0}(x)+a(x)^{-1} u_{1}(x), & x \in \Omega\end{cases}
$$

(which is equivalent to (1.6)) in the sense of

$$
\begin{gathered}
\|\sqrt{a(\cdot)}(u(\cdot, t)-v(\cdot, t))\|_{L^{2}(\Omega)}=O\left(t^{-\frac{N-\alpha}{2(2-\alpha)}-\eta}\right), \\
\|\sqrt{a(\cdot)} v(\cdot, t)\|_{L^{2}(\Omega)}=O\left(t^{-\frac{N-\alpha}{2(2-\alpha)}}\right)
\end{gathered}
$$

as $t \rightarrow \infty$ for some $\eta>0$ (for detail, see [3, 6, 8, 11, 14, 15, 18, 27, 29]). Recently in Sobajima-Wakasugi [24], diffusion phenomena for the slowly decaying initial data has been proved with $a(x)=|x|^{-\alpha}$; note that this result is only valid for the damping with special structure of homogeneous polynomial type. The diffusion phenomena for general damping satisfying the behavior at the spatial infinity (1.8) is open so far.

The consequence of the second application of supersolutions in Theorem 1.2 is diffusion phenomena for (1.7) under the assumption (1.8).

Before stating the result of diffusion phenomena, we provide weighted energy estimates for (1.7).

Theorem 1.4. Assume that $a(x)$ satisfies (1.8) with $\alpha \in[0,1)$ and the pair $\left(u_{0}, u_{1}\right) \in H_{0}^{1}(\Omega) \times L^{2}(\Omega)$ satisfies

$$
E_{0}=\int_{\Omega}\left(\left|\nabla u_{0}(x)\right|^{2}+\left(u_{1}(x)\right)^{2}\right)\langle x\rangle^{2 \sigma+\alpha} d x+\int_{\Omega}\left(u_{0}(x)\right)^{2}\langle x\rangle^{2 \sigma-\alpha} d x<\infty .
$$

with $\sigma \in\left(0, \frac{N-\alpha}{2}\right)$. Let $u$ be a solution of (1.7) (in a weak sense). Then there exists a positive constant $C$ such that

$$
\begin{array}{r}
\sup _{t \geq 0} \int_{\Omega}\left(|\nabla u(x, t)|^{2}+\left(\partial_{t} u(x, t)\right)^{2}\right)\left(1+t+\langle x\rangle^{\alpha}\right)\left(1+t+\langle x\rangle^{2-\alpha}\right)^{\frac{2 \sigma}{2-\alpha}} d x \leq C E_{0} \\
\sup _{t \geq 0} \int_{\Omega}(u(x, t))^{2}\langle x\rangle^{-\alpha}\left(1+t+\langle x\rangle^{2-\alpha}\right)^{\frac{2 \sigma}{2-\alpha}} d x \leq C E_{0}, \\
\int_{0}^{\infty}\left(\int_{\Omega}\left(\partial_{t} u(x, t)\right)^{2}\langle x\rangle^{-\alpha}\left(1+t+\langle x\rangle^{\alpha}\right)\left(1+t+\langle x\rangle^{2-\alpha}\right)^{\frac{2 \sigma}{2-\alpha}} d x\right) d t \leq C E_{0} .
\end{array}
$$


From the estimates (1.10), we already have the energy decay estimate

$$
(1+t)^{1+\frac{2 \sigma}{2-\alpha}} \int_{\Omega}\left(|\nabla u(x, t)|^{2}+\left(\partial_{t} u(x, t)\right)^{2}\right) d x \leq C E_{0} .
$$

Combining (1.12) with the usual energy equality

$$
\int_{\Omega}\left(|\nabla u(x, t)|^{2}+\left(\partial_{t} u(x, t)\right)^{2}\right) d x=2 \int_{t}^{\infty}\left(\int_{\Omega} a(x)\left(\partial_{t} u(x, s)\right)^{2} d x\right) d s
$$

(verified from $\int_{\Omega}\left(|\nabla u|^{2}+\left(\partial_{t} u\right)^{2}\right) d x \rightarrow 0$ as $t \rightarrow \infty$ ), we obtain an energy decay estimate which is slightly stronger than (1.13).

Corollary 1.5. Under the assumption of Theorem 1.4, one has

$$
\lim _{t \rightarrow \infty}\left((1+t)^{1+\frac{2 \sigma}{2-\alpha}} \int_{\Omega}\left(|\nabla u(x, t)|^{2}+\left(\partial_{t} u(x, t)\right)^{2}\right) d x\right)=0 .
$$

The following assertion describes diffusion phenomena for (1.7) with polynomially decaying initial data.

Theorem 1.6. Assume that a $(x)$ satisfies (1.8) and the pair $\left(u_{0}, u_{1}\right) \in\left(H^{2}(\Omega) \cap H_{0}^{1}(\Omega)\right) \times H_{0}^{1}(\Omega)$ satisfies

$$
\begin{aligned}
& E_{0}=\int_{\Omega}\left(\left|\nabla u_{0}(x)\right|^{2}+\left(u_{1}(x)\right)^{2}\right)\langle x\rangle^{2 \sigma+\alpha} d x+\int_{\Omega}\left(u_{0}(x)\right)^{2}\langle x\rangle^{2 \sigma-\alpha} d x<\infty, \\
& E_{0}^{\prime}=\int_{\Omega}\left(\left|\nabla u_{1}(x)\right|^{2}+\left(u_{2}(x)\right)^{2}\right)\langle x\rangle^{2 \sigma+3 \alpha} d x+\int_{\Omega}\left(u_{1}(x)\right)^{2}\langle x\rangle^{2 \sigma+\alpha} d x<\infty
\end{aligned}
$$

with $u_{2}(x)=-\Delta u_{0}(x)+a(x) u_{1}(x)$ and $\sigma \in\left(0, \frac{N-\alpha}{2}\right)$. Let $u$ and $v$ be solutions of (1.7) and (1.9) with $v_{0}(x)=u_{0}(x)+a(x)^{-1} u_{1}(x)$, respectively. Then there exists a positive constant $K$ such that

$$
\|\sqrt{a(\cdot)}(u(x, t)-v(x, t))\|_{L^{2}(\Omega)} \leq K(1+t)^{-\frac{\sigma}{2-\alpha}} \eta(t) \sqrt{E_{0}+E_{0}^{\prime}},
$$

where

$$
\eta(t)= \begin{cases}(1+t)^{-\frac{2(1-\alpha)}{2-\alpha}} \sqrt{\log (2+t)} & \text { if } \sigma \in\left[\alpha, \frac{N-\alpha}{2}\right), \\ (1+t)^{-\frac{2(1-\alpha) \sigma}{(2-\alpha) \alpha}} & \text { if } \sigma \in(0, \alpha) .\end{cases}
$$

Remark 1.3. Since $v_{0}=u_{0}+a(x)^{-1} u_{1}$ (the initial value of $v$ ) satisfies $\langle x\rangle^{\sigma-\frac{\alpha}{2}} v_{0} \in L^{2}(\Omega)$, Proposition 1.3 gives

$$
\|\sqrt{a(\cdot)} v(t)\|_{L^{2}(\Omega)} \leq C(1+t)^{-\frac{\sigma}{2-\alpha}}\left\|\langle x\rangle^{\sigma-\frac{\alpha}{2}} v_{0}\right\|_{L^{2}(\Omega)} .
$$

Therefore Theorem 1.6 enables us to conclude that $v(t)$ provides the asymptotic profile of the solution $u$. In [24, Remark 1.4], a decay estimate similar to (1.14) via weighted $L^{p}-L^{q}$ estimates are provided but with small extra growth factor $(1+t)^{\varepsilon}$. The merit of this procedure via Proposition 1.3 is to reduce unexpected factor $(1+t)^{\varepsilon}$.

As a corollary of Theorem 1.6, we also have a limiting case $\sigma=\frac{N-\alpha}{2}$ as follows. 
Corollary 1.7. Assume that a $(x)$ satisfies (1.8) and the pair $\left(u_{0}, u_{1}\right) \in\left(H^{2}(\Omega) \cap H_{0}^{1}(\Omega)\right) \times H_{0}^{1}(\Omega)$ satisfies

$$
\langle x\rangle^{\frac{N}{2}} u_{0},\langle x\rangle^{\frac{N}{2}+\alpha} \nabla u_{0},\langle x\rangle^{\frac{N}{2}+\alpha} u_{1},\langle x\rangle^{\frac{N}{2}+2 \alpha} \Delta u_{0},\langle x\rangle^{\frac{N}{2}+2 \alpha} \nabla u_{1} \in L^{2}(\Omega) .
$$

Let $u$ and $v$ be solutions of (1.7) and (1.9) with $v_{0}(x)=u_{0}(x)+a(x)^{-1} u_{1}(x)$, respectively. Then for every $\varepsilon>0$, there exists a positive constant $K_{\varepsilon}$ such that

$$
\|\sqrt{a(\cdot)}(u(\cdot, t)-v(\cdot, t))\|_{L^{2}(\Omega)} \leq K_{\varepsilon}(1+t)^{-\frac{N-\alpha}{2(2-\alpha)}-\frac{2(1-\alpha)}{2-\alpha}+\varepsilon} .
$$

Remark 1.4. Energy estimates with polynomial growth weights (such as Theorem 1.4) can be also applied to semilinear wave equations with damping term $a(x) \partial_{t} u$ (for example, see Sobajima [20] for the case $a(x) \equiv 1$ ). This kind of analysis including asymptotic behavior of solutions to nonlinear problem will be done in a forthcoming paper.

The present paper is organized as follows. In Section 2, the basic properties of Kummer's confluent hypergeometric functions are collected, which are deeply used throughout of this paper. Section 3 is devoted to prove Theorem 1.2, that is, the construction of supersolution to $\partial_{t} U-D(x) \Delta U=0$. As applications, we will prove weighted $L^{2}$-type decay estimates for initial-boundary problem of $\partial_{t} v-$ $D(x) \Delta v=0$ via semigroup approach in Section 4. In the last Section 5, we show weighted energy estimates for solutions to wave equations with damping term $\partial_{t}^{2} u-\Delta u+a(x) \partial_{t} u=0$ and their diffusion phenomena.

\section{Preliminaries}

We collect some of important properties of Kummer's confluent hypergeometric functions. At the beginning we state their definition.

Definition 2.1 (Kummer's confluent hypergeometric functions). For $b, c \in \mathbb{R}$ with $-c \notin \mathbb{N} \cup\{0\}$, Kummer's confluent hypergeometric function of first kind is defined by

$$
M(b, c ; s)=\sum_{n=0}^{\infty} \frac{(b)_{n}}{(c)_{n}} \frac{s^{n}}{n !}, \quad s \in[0, \infty),
$$

where $(d)_{n}$ is the Pochhammer symbol defined by $(d)_{0}=1$ and $(d)_{n}=\prod_{k=1}^{n}(d+k-1)$ for $n \in \mathbb{N}$; note that when $b=c$, Kummer's function $M(b, b ; s)$ coincides with $e^{s}$.

The following properties of Kummer's confluent hypergeometric functions are well known (see e.g., Beals-Wong [1]).

Lemma 2.2. Kummer's confluent hypergeometric function $M(b, c ; s)$ satisfies the following properties:

(i) $M(b, c ; s)$ is a solution of the Kummer equation

$$
s u(s)^{\prime \prime}+(c-s) u^{\prime}(s)-b u(s)=0 .
$$

(ii) If $c \geq b>0$, then $M(b, c ; s)>0$ and $M(b, c ; s) \sim \frac{\Gamma(c)}{\Gamma(b)} s^{b-c} e^{s}$ as $s \rightarrow \infty$, more precisely,

$$
\lim _{s \rightarrow \infty} \frac{M(b, c ; s)}{s^{b-c} e^{s}}=\frac{\Gamma(c)}{\Gamma(b)}
$$


(iii) More generally, if $-c \notin \mathbb{N} \cap\{0\}$ and $c \geq b$, then, while the sign of $M(b, c ; s)$ is indefinite, it has the same asymptotic behavior

$$
\lim _{s \rightarrow \infty} \frac{M(b, c ; s)}{s^{b-c} e^{s}}=\frac{\Gamma(c)}{\Gamma(b)} .
$$

In particular, $M(b, c ; s)$ has a bound

$$
|M(b, c ; s)| \leq C_{b, c}(1+s)^{b-c} e^{s}
$$

with some constant $C_{b, c}>0$.

(iv) $M(b, c ; s)$ satisfies the relations

$$
\begin{aligned}
s M(b, c ; s) & =s M^{\prime}(b, c ; s)+(c-b) M(b, c ; s)-(c-b) M(b-1, c ; s), \\
c M^{\prime}(b, c ; s) & =c M(b, c ; s)-(c-b) M(b, c+1 ; s) .
\end{aligned}
$$

Remark 2.1. For the proof of (i), (ii), and (iv), see [1, p.190, (6.1.1)], [1, p.192, (6.1.8)], and [1, p.200], respectively. The assertion (iii) can be found in [1, p.192, the comment under (6.1.9)]. Also, we easily prove it in the following way. We first note that

$$
M^{(m)}(b, c ; s)=\frac{(b)_{m}}{(c)_{m}} M(b+m, c+m ; s)
$$

and $\left|M^{(m)}(b, c ; s)\right| \rightarrow \infty$ as $s \rightarrow \infty$ for any $m \in \mathbb{Z}_{\geq 0}$. Let $m \in \mathbb{Z}_{\geq 0}$ be such that $b+m>0$ holds. Then, the l'Hôpital theorem and (ii) imply

$$
\lim _{s \rightarrow \infty} \frac{M(b, c ; s)}{s^{b-c} e^{s}}=\lim _{s \rightarrow \infty} \frac{D_{s}^{m} M(b, c ; s)}{D_{s}^{m}\left(s^{b-c} e^{s}\right)}=\frac{(b)_{m}}{(c)_{m}} \lim _{s \rightarrow \infty} \frac{M(b+m, c+m ; s)}{s^{b-c} e^{s}+o\left(s^{b-c} e^{s}\right)}=\frac{(b)_{m}}{(c)_{m}} \frac{\Gamma(c+m)}{\Gamma(b+m)}=\frac{\Gamma(c)}{\Gamma(b)} .
$$

\section{Construction of supersolution to $\partial_{t} U=D(x) \Delta U$}

In this section, we will construct a family of positive supersolutions to the parabolic equation

$$
\partial_{t} U-D(x) \Delta U=0, \quad(x, t) \in \Omega \times(0, \infty) .
$$

Here we assume that $\alpha \in(-\infty, \min \{2, N\})$ in (1.2). Following the previous works [21, 22, 23], we use the same notation as the damping coefficient $a(x)$. Therefore we put

$$
a(x):=\frac{1}{D(x)} .
$$

Since $D(x)$ is positive, the problem (1.1) is equivalent to

$$
a(x) \partial_{t} U(x, t)-\Delta U(x, t)=0, \quad(x, t) \in \Omega \times(0, \infty) .
$$

Also, the assumption (1.2) implies that

$$
\lim _{|x| \rightarrow \infty}\left(|x|^{\alpha} a(x)\right)=\frac{1}{D_{0}}>0 .
$$


To fix the direction of the discussion, we first recall the special case where $a(x)=|x|^{-\alpha}\left(D(x)=|x|^{\alpha}\right)$ with $\alpha \in[0,1)$, which is studied in [24, Section 2]. In this case, the equation (3.1) becomes

$$
|x|^{-\alpha} \partial_{t} U=\Delta U, \quad(x, t) \in \mathbb{R}^{N} \times(0, \infty) .
$$

This equation has a self-similar structure. Indeed, if $U(x, t)$ is the solution of $(3.5)$, then $U_{s}(x, t)=$ $U\left(s^{\frac{1}{2-\alpha}} x, s t\right)$ is also a solution of the same equation. Therefore, we can introduce a notion of self-similar solutions of the type $U=s^{\beta} U_{s}(\beta>0)$. Fortunately, such a family of solutions can be explicitly written by using Kummer's confluent hypergeometric functions. (In this moment, to use this kind of self-similar structure we shall impose $\alpha<2$. The other cases can be considered via the Kelvin transform.) The following lemma is the list of their important properties.

Lemma 3.1 ([24, Section 2]). For $\beta>0$ and $\alpha \in[0,1)$, define

$$
\widetilde{\Phi}_{\beta}(x, t)=t^{-\beta} \varphi_{\beta}(\xi(x, t)), \quad \varphi_{\beta}(z)=e^{-z} M\left(\frac{N-\alpha}{2-\alpha}-\beta, \frac{N-\alpha}{2-\alpha} ; z\right), \quad \xi(x, t)=\frac{|x|^{2-\alpha}}{(2-\alpha)^{2} t} .
$$

Then $\widetilde{\Phi}_{\beta} \in C^{\infty}\left(\mathbb{R}^{N} \times(0, \infty)\right)$ satisfies the following assertions:

(i) for every $\beta>0$ and $s>0$,

$$
\widetilde{\Phi}_{\beta}(x, t)=s^{\beta} \widetilde{\Phi}_{\beta}\left(s^{\frac{1}{2-\alpha}} x, s t\right), \quad(x, t) \in \mathbb{R}^{N} \times(0, \infty) ;
$$

(ii) for every $\beta>0$,

$$
|x|^{-\alpha} \partial_{t} \widetilde{\Phi}_{\beta}(x, t)=\Delta \widetilde{\Phi}_{\beta}(x, t), \quad(x, t) \in \mathbb{R}^{N} \times(0, \infty) ;
$$

(iii) for every $\beta>0$, there exists a positive constant $C_{\beta}>0$ such that

$$
\left|\widetilde{\Phi}_{\beta}(x, t)\right| \leq C_{\beta}\left(t+\frac{|x|^{2-\alpha}}{(2-\alpha)^{2}}\right)^{-\beta}, \quad(x, t) \in \mathbb{R}^{N} \times(0, \infty) ;
$$

(iv) for every $0<\beta<\frac{N-\alpha}{2-\alpha}$, there exists a positive constant $c_{\beta}>0$ such that

$$
\widetilde{\Phi}_{\beta}(x, t) \geq c_{\beta}\left(t+\frac{|x|^{2-\alpha}}{(2-\alpha)^{2}}\right)^{-\beta}, \quad(x, t) \in \mathbb{R}^{N} \times(0, \infty) ;
$$

(v) for every $\beta>0$,

$$
\partial_{t} \widetilde{\Phi}_{\beta}(x, t)=-\beta \widetilde{\Phi}_{\beta+1}(x, t), \quad(x, t) \in \mathbb{R}^{N} \times(0, \infty) .
$$

The aim of this section is to construct a family of supersolutions to (3.1) of the form

$$
\left(t_{0}+t\right)^{-\beta} \varphi\left(\frac{\gamma A(x)}{t_{0}+t}\right)
$$

with properties similar to Lemma 3.1 (iii)-(v), which is motivated by the family $\left\{\widetilde{\Phi}_{\beta}\right\}_{\beta>0}$ in Lemma 3.1. 


\subsection{Related elliptic problem}

Observe that the relation

$$
\Delta\left(\frac{|x|^{2-\alpha}}{(2-\alpha)^{2}}\right)=\frac{N-\alpha}{2-\alpha}|x|^{-\alpha}=\text { (const.) } a(x)
$$

seems to be important. As the generalization of this relation, we recall existence of approximate solutions of the Poisson equation

$$
\Delta A(x)=a(x), \quad x \in \mathbb{R}^{N}
$$

in the sense (3.7) below. We refer [22, Lemma 2.1] for $\alpha \in[0,1)$, [23, Lemma 3.1] for $\alpha \in(-\infty, 0)$ for the proof. The proof of the remaining cases $\alpha \in[1,2)$ is essentially the same as [22, Lemma 2.1].

Lemma 3.2. Assume that $a(x)$ satisfies (3.4) with $\alpha \in(-\infty, \min \{2, N\})$. Then for every $\varepsilon \in(0,1)$, there exist a function $A_{\varepsilon} \in C^{2}\left(\mathbb{R}^{N}\right)$ and positive constants $c_{\varepsilon}$ and $C_{\varepsilon}$ such that

$$
\begin{aligned}
& (1-\varepsilon) a(x) \leq \Delta A_{\varepsilon}(x) \leq(1+\varepsilon) a(x), \\
& c_{\varepsilon}\langle x\rangle^{2-\alpha} \leq A_{\varepsilon}(x) \leq C_{\varepsilon}\langle x\rangle^{2-\alpha}, \\
& \frac{\left|\nabla A_{\varepsilon}(x)\right|^{2}}{a(x) A_{\varepsilon}(x)} \leq \frac{2-\alpha}{N-\alpha}+\varepsilon
\end{aligned}
$$

hold for $x \in \mathbb{R}^{N}$.

Remark 3.1. It is enough to find an exact solution of (3.6) satisfying (3.8) and (3.9) to construct a supersolution of parabolic equation (3.1). If $a(x)$ is radially symmetric, the solution $A$ of Poisson equation was constructed in Todorova-Yordanov [25]. In subsequent papers, suitable profiles for more general problem, such as damped wave equation with space-dependent diffusion, have been considered (see e.g., Radu-Todorova-Yordanov [16, 17]). However, such a solution with expected properties does not exist in general (especially in the case where $a(x)$ is not radially symmetric, see [21, Remark 3.1]).

\subsection{Supersolution of corresponding parabolic equation}

For a function $\varphi \in C^{2}([0, \infty))$ and $(x, t) \in \mathbb{R}^{N} \times[0, \infty)$, we put

$$
\Phi(x, t)=\left(t_{0}+t\right)^{-\beta} \varphi(z), \quad z=\frac{\gamma A_{\varepsilon}(x)}{t_{0}+t}
$$

with some constants $\beta, \gamma>0$ and $t_{0} \geq 1$. Here, $A_{\varepsilon}(x)$ is the function constructed in Lemma 3.2 with a constant $\varepsilon \in(0,1)$. We will specify the function $\varphi$ later, and here we first show the following lemma.

Lemma 3.3. Let $\varphi \in C^{2}([0, \infty))$ and $\Phi$ be as in (3.10). Then

$$
a(x) \partial_{t} \Phi(x, t)-\Delta \Phi(x, t)=-a(x)\left(t_{0}+t\right)^{-\beta-1}\left(\beta \varphi(z)+z \varphi^{\prime}(z)+\gamma \frac{\Delta A_{\varepsilon}(x)}{a(x)} \varphi^{\prime}(z)+\gamma \frac{\left|\nabla A_{\varepsilon}(x)\right|^{2}}{a(x) A_{\varepsilon}(x)} z \varphi^{\prime \prime}(z)\right)
$$

holds for $(x, t) \in \mathbb{R}^{N} \times[0, \infty)$. 
Proof. By direct calculation we have

$$
a(x) \partial_{t} \Phi(x, t)=a(x)\left(t_{0}+t\right)^{-\beta-1}\left(-\beta \varphi(z)-z \varphi^{\prime}(z)\right),
$$

where $z=\gamma A_{\varepsilon}(x) /\left(t_{0}+t\right)$. On the one hand, we see that

$$
\begin{aligned}
\Delta \Phi(x, t) & =\left(t_{0}+t\right)^{-\beta}\left(\gamma \frac{\Delta A_{\varepsilon}(x)}{t_{0}+t} \varphi^{\prime}(z)+\gamma^{2} \frac{\left|\nabla A_{\varepsilon}(x)\right|^{2}}{\left(t_{0}+t\right)^{2}} \varphi^{\prime \prime}(z)\right) \\
& =a(x)\left(t_{0}+t\right)^{-\beta-1}\left(\gamma \frac{\Delta A_{\varepsilon}(x)}{a(x)} \varphi^{\prime}(z)+\frac{\gamma\left|\nabla A_{\varepsilon}(x)\right|^{2}}{a(x) A_{\varepsilon}(x)} z \varphi^{\prime \prime}(z)\right) .
\end{aligned}
$$

Therefore we have (3.11).

In particular, we choose

$$
\widetilde{\gamma}_{\varepsilon}=\left(\frac{2-\alpha}{N-\alpha}+\varepsilon\right)^{-1}, \quad \gamma_{\varepsilon}=(1-\varepsilon) \widetilde{\gamma}_{\varepsilon} .
$$

and $\varphi$ by using the Kummer confluent hypergeometric function.

Definition 3.4. For $\beta \in \mathbb{R}$, define

$$
\varphi_{\beta, \varepsilon}(s)=e^{-s} M\left(\gamma_{\varepsilon}-\beta, \gamma_{\varepsilon} ; s\right), \quad s \geq 0 .
$$

We remark that $\varphi_{\beta, \varepsilon}$ is a unique (modulo constant multiple) solution of the equation

$$
s \varphi^{\prime \prime}(s)+\left(\gamma_{\varepsilon}+s\right) \varphi^{\prime}(s)+\beta \varphi(s)=0
$$

with bounded derivative near $s=0$.

Then we have the following properties of $\varphi_{\beta, \varepsilon}$.

Lemma 3.5. The function $\varphi_{\beta, \varepsilon}$ satisfies the following:

(i) If $\beta \in\left[0, \gamma_{\varepsilon}\right)$, then $\varphi_{\beta, \varepsilon}$ has the lower and upper bounds

$$
k_{\beta, \varepsilon}(1+s)^{-\beta} \leq \varphi_{\beta, \varepsilon}(s) \leq K_{\beta, \varepsilon}(1+s)^{-\beta}
$$

with some constants $k_{\beta, \varepsilon}, K_{\beta, \varepsilon}>0$.

(ii) If $\beta \geq 0$, then $\varphi_{\beta, \varepsilon}$ has the upper bound

$$
\left|\varphi_{\beta, \varepsilon}(s)\right| \leq K_{\beta, \varepsilon}(1+s)^{-\beta}
$$

with some constant $K_{\beta, \varepsilon}>0$.

(iii) If $\beta \geq 0$, then $\varphi_{\beta, \varepsilon}(s)$ and $\varphi_{\beta+1, \varepsilon}(s)$ satisfy the recurrence relation

$$
\beta \varphi_{\beta, \varepsilon}(s)+s \varphi_{\beta, \varepsilon}^{\prime}(s)=\beta \varphi_{\beta+1, \varepsilon}(s) .
$$

(iv) If $0<\beta<\gamma_{\varepsilon}$, the first and the second derivatives of $\varphi_{\beta, \varepsilon}(s)$ have negative and positive signs, respectively:

$$
\varphi_{\beta, \varepsilon}^{\prime}(s)=-\frac{\beta}{\gamma_{\varepsilon}} e^{-s} M\left(\gamma_{\varepsilon}-\beta, \gamma_{\varepsilon}+1 ; s\right)<0, \quad \varphi_{\beta, \varepsilon}^{\prime \prime}(s)=\frac{\beta(\beta+1)}{\gamma_{\varepsilon}\left(\gamma_{\varepsilon}+1\right)} e^{-s} M\left(\gamma_{\varepsilon}-\beta, \gamma_{\varepsilon}+2 ; s\right)>0 .
$$


Proof. (i) From Lemma 2.2 (ii), we obtain

$$
k_{\beta, \varepsilon} e^{s}(1+s)^{-\beta} \leq M\left(\gamma_{\varepsilon}-\beta, \gamma_{\varepsilon} ; s\right) \leq K_{\beta, \varepsilon} e^{s}(1+s)^{-\beta}
$$

for $s \geq 0$ with some positive constants $k_{\beta, \varepsilon}, K_{\beta, \varepsilon}$. This implies (i).

(ii) Similarly to (i), Lemma 2.2 (iii) implies

$$
\left|M\left(\gamma_{\varepsilon}-\beta, \gamma_{\varepsilon} ; s\right)\right| \leq K_{\beta, \varepsilon}(1+s)^{-\beta} e^{s} .
$$

This and the definition of $\varphi_{\beta, \varepsilon}$ lead to (ii).

(iii) Noting

$$
\varphi_{\beta, \varepsilon}^{\prime}(s)=e^{-s}\left(-M\left(\gamma_{\varepsilon}-\beta, \gamma_{\varepsilon} ; s\right)+M^{\prime}\left(\gamma_{\varepsilon}-\beta, \gamma_{\varepsilon} ; s\right)\right)
$$

and the first assertion of Lemma 2.2 (iv), we have

$$
\begin{aligned}
& \beta \varphi_{\beta, \varepsilon}(s)+s \varphi_{\beta, \varepsilon}^{\prime}(s) \\
& =e^{-s}\left(\beta M\left(\gamma_{\varepsilon}-\beta, \gamma_{\varepsilon} ; s\right)-s M\left(\gamma_{\varepsilon}-\beta, \gamma_{\varepsilon} ; s\right)+s M^{\prime}\left(\gamma_{\varepsilon}-\beta, \gamma_{\varepsilon} ; s\right)\right) \\
& =\beta e^{-s} M\left(\gamma_{\varepsilon}-\beta-1, \gamma_{\varepsilon} ; s\right) \\
& =\beta \varphi_{\beta+1, \varepsilon}(s) .
\end{aligned}
$$

(iv) The second assertion of Lemma 2.2 (iv) implies

$$
\gamma_{\varepsilon} M^{\prime}\left(\gamma_{\varepsilon}-\beta, \gamma_{\varepsilon} ; s\right)=\gamma_{\varepsilon} M\left(\gamma_{\varepsilon}-\beta, \gamma_{\varepsilon} ; s\right)-\beta M\left(\gamma_{\varepsilon}-\beta, \gamma_{\varepsilon}+1 ; s\right) .
$$

From this and (3.14), we obtain

$$
\varphi_{\beta, \varepsilon}^{\prime}(s)=-\frac{\beta}{\gamma_{\varepsilon}} e^{-s} M\left(\gamma_{\varepsilon}-\beta, \gamma_{\varepsilon}+1 ; s\right) .
$$

Since $0<\beta<\gamma_{\varepsilon}$, Lemma 2.2 (ii) shows $M\left(\gamma_{\varepsilon}-\beta, \gamma_{\varepsilon}+1 ; s\right)>0$ and hence, $\varphi_{\beta, \varepsilon}^{\prime}(s)<0$.

Next, we compute

$$
\varphi_{\beta, \varepsilon}^{\prime \prime}(s)=-\frac{\beta}{\gamma_{\varepsilon}} e^{-s}\left(-M\left(\gamma_{\varepsilon}-\beta, \gamma_{\varepsilon}+1 ; s\right)+M^{\prime}\left(\gamma_{\varepsilon}-\beta, \gamma_{\varepsilon}+1 ; s\right)\right) .
$$

Applying the second assertion of Lemma 2.2 (iv), we have

$$
\left(\gamma_{\varepsilon}+1\right) M^{\prime}\left(\gamma_{\varepsilon}-\beta, \gamma_{\varepsilon}+1 ; s\right)=\left(\gamma_{\varepsilon}+1\right) M\left(\gamma_{\varepsilon}-\beta, \gamma_{\varepsilon}+1 ; s\right)-(\beta+1) M\left(\gamma_{\varepsilon}-\beta, \gamma_{\varepsilon}+2 ; s\right),
$$

and hence,

$$
\varphi_{\beta, \varepsilon}^{\prime \prime}(s)=\frac{\beta(\beta+1)}{\gamma_{\varepsilon}\left(\gamma_{\varepsilon}+1\right)} e^{-s} M\left(\gamma_{\varepsilon}-\beta, \gamma_{\varepsilon}+2 ; s\right) .
$$

Noting again that $0<\beta<\gamma_{\varepsilon}$, we see from Lemma 2.2 (ii) that $M\left(\gamma_{\varepsilon}-\beta, \gamma_{\varepsilon}+2 ; s\right)>0$, and hence, $\varphi_{\beta, \varepsilon}^{\prime \prime}(s)>0$. 1.2

Here we define a family of functions $\left\{\Phi_{\beta, \varepsilon}\right\}_{\beta}$ which will be proved to fulfill all conditions in Theorem 
Definition 3.6. For $(x, t) \in \mathbb{R}^{N} \times[0, \infty)$, we define

$$
\Phi_{\beta, \varepsilon}\left(x, t ; t_{0}\right)=\left(t_{0}+t\right)^{-\beta} \varphi_{\beta, \varepsilon}(z), \quad z=\frac{\widetilde{\gamma}_{\varepsilon} A_{\varepsilon}(x)}{t_{0}+t},
$$

where $\varepsilon \in(0,1), \widetilde{\gamma}_{\varepsilon}$ is the constant given in (3.12), $\beta$ is a constant satisfying $\beta \in\left(0, \widetilde{\gamma_{\varepsilon}}\right), t_{0} \geq 1, \varphi_{\beta, \varepsilon}$ is the function defined by Definition 3.4, and $A_{\varepsilon}(x)$ is the function constructed in Lemma 3.2.

The function $\Phi_{\beta, \varepsilon}(x, t)$ defined above is a supersolution of the equation (3.3).

Lemma 3.7. The function $\Phi_{\beta, \varepsilon}\left(x, t ; t_{0}\right)$ satisfies

$$
a(x) \partial_{t} \Phi_{\beta, \varepsilon}\left(x, t ; t_{0}\right)-\Delta \Phi_{\beta, \varepsilon}\left(x, t ; t_{0}\right) \geq 0, \quad(x, t) \in \mathbb{R}^{N} \times[0, \infty) .
$$

Proof. We note that the equation (3.13) implies

$$
\begin{aligned}
\beta \varphi_{\beta, \varepsilon}(z)+z \varphi_{\beta, \varepsilon}^{\prime}(z) & =-\gamma_{\varepsilon} \varphi_{\beta, \varepsilon}^{\prime}(z)-z \varphi_{\beta, \varepsilon}^{\prime \prime}(z) \\
& =-(1-\varepsilon){\widetilde{\gamma_{\varepsilon}}}_{\beta, \varepsilon}^{\prime}(z)-z \varphi_{\beta, \varepsilon}^{\prime \prime}(z) .
\end{aligned}
$$

Using the above and Lemma 3.3, we calculate

$$
\begin{aligned}
& a(x) \partial_{t} \Phi_{\beta, \varepsilon}(x, t)-\Delta \Phi_{\beta, \varepsilon}(x, t) \\
& =-a(x)\left(t_{0}+t\right)^{-\beta-1}\left(\beta \varphi_{\beta, \varepsilon}(z)+z \varphi_{\beta, \varepsilon}^{\prime}(z)+\widetilde{\gamma}_{\varepsilon} \frac{\Delta A_{\varepsilon}(x)}{a(x)} \varphi_{\beta, \varepsilon}^{\prime}(z)+\widetilde{\gamma}_{\varepsilon} \frac{\left|\nabla A_{\varepsilon}(x)\right|^{2}}{a(x) A_{\varepsilon}(x)} z \varphi_{\beta, \varepsilon}^{\prime \prime}(z)\right) \\
& =\widetilde{\gamma}_{\varepsilon} a(x)\left(t_{0}+t\right)^{-\beta-1}\left(1-\varepsilon-\frac{\Delta A_{\varepsilon}(x)}{a(x)}\right) \varphi_{\beta, \varepsilon}^{\prime}(z)+a(x)\left(t_{0}+t\right)^{-\beta-1} z\left(1-\widetilde{\gamma}_{\varepsilon} \frac{\left|\nabla A_{\varepsilon}(x)\right|^{2}}{a(x) A_{\varepsilon}(x)}\right) \varphi_{\beta, \varepsilon}^{\prime \prime}(z) .
\end{aligned}
$$

Since it follows from the construction of $A_{\varepsilon}$ that

$$
1-\varepsilon-\frac{\Delta A_{\varepsilon}(x)}{a(x)} \leq 0, \quad 1-\widetilde{\gamma}_{\varepsilon} \frac{\left|\nabla A_{\varepsilon}(x)\right|^{2}}{a(x) A_{\varepsilon}(x)} \geq 0
$$

we see from Lemma 3.5 (iii) that $\Phi_{\beta, \varepsilon}$ satisfies $a(x) \partial_{t} \Phi_{\beta, \varepsilon}(x, t)-\Delta \Phi_{\beta, \varepsilon}(x, t) \geq 0$ for all $x \in \mathbb{R}^{N}$ and $t \geq 0$.

The function $\Phi_{\beta, \varepsilon}$ also satisfies the following recurrence relation.

Lemma 3.8. Let $\Phi_{\beta, \varepsilon}$ be defined in Definition 3.6. Then, for $\beta \geq 0$, we have

$$
\partial_{t} \Phi_{\beta, \varepsilon}\left(t ; t_{0}\right)=-\beta \Phi_{\beta+1, \varepsilon}\left(t ; t_{0}\right) .
$$

The lemma above immediately follows from Lemma 3.5 (iii) and we omit the detail.

Proof of Theorem 1.2. Put $\beta=\frac{2 \sigma}{2-\alpha}$ and take $\varepsilon>0$ sufficiently small so that $\beta<\widetilde{\gamma}_{\varepsilon}$, and let $t_{0} \geq 1$. We define

$$
\bar{U}_{D, \sigma}(x, t)=\widetilde{K} \Phi_{\beta, \varepsilon}\left(x, t ; t_{0}\right), \quad(x, t) \in \Omega \times[0, \infty),
$$

where $\widetilde{K}$ is a positive constant specified later. Then Lemma 3.7 yields that $\Phi_{\beta, \varepsilon}\left(x, t ; t_{0}\right)$ is a supersolution of $a(x) \partial_{t} U-\Delta U=0$ in $\mathbb{R}^{N}$. Concerning the initial value and the boundary condition, we see from Lemma 3.5 (i) and Lemma 3.2 that

$$
\widetilde{K} k_{\beta, \varepsilon}\left(t_{0}+t+C_{\varepsilon}\langle x\rangle^{2-\alpha}\right)^{-\frac{2 \sigma}{2-\alpha}} \leq \bar{U}_{D, \sigma}(x, t) \leq \widetilde{K} K_{\beta, \varepsilon}\left(t_{0}+t+c_{\varepsilon}\langle x\rangle^{2-\alpha}\right)^{-\frac{2 \sigma}{2-\alpha}} .
$$

Taking $\widetilde{K}=k_{\beta, \varepsilon}^{-1}\left(t_{0}+C_{\varepsilon}\right)^{\frac{2 \sigma}{2-\alpha}}$, we have $\bar{U}_{D, \sigma}(x, 0) \geq\langle x\rangle^{-2 \sigma}=w(x)$ for $x \in \Omega$. Since $\Phi_{\beta, \varepsilon}\left(x, t ; t_{0}\right)$ is positive in $\mathbb{R}^{N} \times[0, \infty), \bar{U}_{D, \sigma}$ satisfies the nonnegativity condition on $\partial \Omega \times[0, \infty)$. As a consequence, $\bar{U}_{D, \sigma}$ is a supersolution of the problem (1.1). The estimate (1.4) follows from (3.15) and the other estimate (1.5) follows from Lemma 3.8 and Lemma 3.5 (ii). The proof is now complete. 


\section{Application to weighted $L^{2}$-estimates for diffusion equations}

In this section, as an application of supersolutions to (1.1) in Theorem 1.2, we discuss some weighted $L^{2}$ estimates for the initial-boundary value problem of the linear diffusion equation (1.6) which is equivalent to the following problem in terms of $a(x)=D(x)^{-1}$ :

$$
\begin{cases}a(x) \partial_{t} u(x, t)-\Delta u(x, t)=0, & (x, t) \in \Omega \times(0, \infty) \\ u(x, t)=0, & (x, t) \in \partial \Omega \times(0, \infty), \\ u(0, x)=f(x), & x \in \Omega .\end{cases}
$$

Here, $\Omega$ is an exterior domain in $\mathbb{R}^{N}$ with smooth boundary $\partial \Omega$ with $N \geq 2$, namely, $\mathbb{R}^{N} \backslash \Omega$ is compact. The operator $a(x)^{-1} \Delta$ is formally symmetric in the following Hilbert space $L_{d \mu}^{2}=L_{d \mu}^{2}(\Omega)$ with the inner product $(\cdot, \cdot)_{L_{d \mu}^{2}}$ :

$$
L_{d \mu}^{2}(K):=\left\{f \in L_{\mathrm{loc}}^{2}(K) ;\|f\|_{L_{d \mu}^{2}(K)}=\left(\int_{K}|f|^{2} d \mu\right)^{\frac{1}{2}}<\infty\right\}, \quad(f, g)_{L_{d \mu}^{2}(K)}=\int_{K} f g d \mu, \quad d \mu=a(x) d x .
$$

According to the analysis of [21, Section 2], the corresponding bilinear closed form is given by

$$
\mathfrak{a}(u, v)=\int_{\Omega} \nabla u \cdot \nabla v d x, \quad D(\mathfrak{a})=\left\{u \in L_{d \mu}^{2} \cap \dot{H}^{1}(\Omega) ; \int_{\Omega} \frac{\partial u}{\partial x_{j}} \varphi d x=-\int_{\Omega} u \frac{\partial \varphi}{\partial x_{j}}, \forall \varphi \in C_{c}^{\infty}\left(\mathbb{R}^{N}\right)\right\} .
$$

Note that a similar proof to [21, Lemma 2.1] works for $\alpha<2$. Then we can use the Friedrichs extension $-L$ of $-a(x) \Delta$ in $L_{d \mu}^{2}$ as the associated operator of the closed form $\mathfrak{a}$.

Lemma 4.1 ([21, Lemma 2.2]). The operator $L$ in $L_{d \mu}^{2}$ defined by

$$
\begin{aligned}
D(L) & =\left\{u \in D(\mathfrak{a}) ; \exists f \in L_{d \mu}^{2} \text { s.t. } \mathfrak{a}(u, v)=(f, v)_{L_{d \mu}^{2}} \quad \forall v \in D(\mathfrak{a})\right\}, \\
-L u & =f
\end{aligned}
$$

is nonnegative and selfadjoint in $L_{d \mu}^{2}$. Therefore $L$ generates an analytic semigroup $T(t)$ on $L_{d \mu}^{2}$ and satisfies

$$
\|T(t) f\|_{L_{d \mu}^{2}} \leq\|f\|_{L_{d \mu}^{2}}, \quad\|L T(t) f\|_{L_{d \mu}^{2}} \leq \frac{1}{t}\|f\|_{L_{d \mu}^{2}} \quad \forall f \in L_{d \mu}^{2} .
$$

Although $L^{p}-L^{q}$ type estimates for the semigroup $T(t)$ are proved in [21] for $\alpha \in(0,1)$ and [23] for $\alpha \in(-\infty, 0)$, we shall provide other type decay estimates. The main assertion of this section is Proposition 1.3 which is rewritten in the following way via the notation in this section.

Proposition 4.2. Let $\sigma \in\left(0, \frac{N-\alpha}{2}\right)$. If $f \in L_{d \mu}^{2}$ satisfies $\langle x\rangle^{\sigma} f \in L_{d \mu}^{2}$, then

$$
\|T(t) f\|_{L_{d \mu}^{2}} \leq C(1+t)^{-\frac{\sigma}{2-\alpha}}\left\|\langle x\rangle^{\sigma} f\right\|_{L_{d \mu}^{2}} \quad \forall t \geq 0 .
$$

Proof. We introduce the following auxiliary problem in $\Omega_{n}=\Omega \cap B(0, n)$, which is an approximation of the original problem (4.1):

$$
\begin{cases}a(x) \partial_{t} u(x, t)-\Delta u(x, t)=0, & (x, t) \in \Omega_{n} \times(0, \infty) \\ u(x, t)=0, & (x, t) \in \partial \Omega_{n} \times(0, \infty), \\ u(0, x)=f(x), & x \in \Omega_{n}\end{cases}
$$


It is sufficient to consider only the case where $\partial \Omega \cap \partial B(0, n)=\emptyset$. By the same procedure as the case of $L$, we have the corresponding generator $L_{n}$ and the semigroup $T_{n}(t)$ satisfying $D\left(L_{n}\right)=H^{2}\left(\Omega_{n}\right) \cap H_{0}^{1}\left(\Omega_{n}\right)$ with

$$
\left\|T_{n}(t) f\right\|_{L_{d \mu}^{2}\left(\Omega_{n}\right)} \leq\|f\|_{L_{d \mu}^{2}\left(\Omega_{n}\right)}, \quad\left\|L_{n} T_{n}(t) g\right\|_{L_{d \mu}^{2}\left(\Omega_{n}\right)} \leq \frac{1}{t}\|g\|_{L_{d \mu}^{2}\left(\Omega_{n}\right)} \quad \forall g \in L_{d \mu}^{2}\left(\Omega_{n}\right)
$$

Now we define the semigroup $\left\{\widetilde{T}_{n}(t)\right\}_{t \geq 0}$ in $L_{d \mu}^{2}$ by

$$
\widetilde{T}_{n}(t) f= \begin{cases}w_{n}(t)=T_{n}(t)\left[\left.f\right|_{\Omega_{n}}\right](x) & x \in \Omega_{n}, \\ f(x) & x \in \Omega \backslash \Omega_{n} .\end{cases}
$$

Here we choose $\beta=\frac{2 \sigma}{2-\alpha}$. Since $\Phi_{\beta, \varepsilon}=\Phi_{\beta, \varepsilon}(\cdot, t ; 1) \in C^{2}\left(\overline{\Omega_{n}}\right)$ is positive, we can verify the following computation:

$$
\begin{aligned}
\frac{d}{d t} \int_{\Omega_{n}}\left|w_{n}(t)\right|^{2} \Phi_{\beta, \varepsilon}(t ; 1)^{-1} d \mu & =2 \int_{\Omega_{n}} w_{n}(t) \partial_{t} w_{n}(t) \Phi_{\beta, \varepsilon}^{-1} d \mu-\int_{\Omega_{n}}\left|w_{n}(t)\right|^{2} \Phi_{\beta, \varepsilon}^{-2} \partial_{t} \Phi_{\beta, \varepsilon} d \mu \\
& =2 \int_{\Omega_{n}} \widetilde{w_{n}}(t) \Delta\left(\widetilde{w_{n}} \Phi_{\beta, \varepsilon}\right) d x-\int_{\Omega_{n}}\left|\widetilde{w_{n}}(t)\right|^{2} a \partial_{t} \Phi_{\beta, \varepsilon} d x \\
& =-2 \int_{\Omega_{n}}\left|\nabla \widetilde{w_{n}}(t)\right|^{2} \Phi_{\beta, \varepsilon} d x-\int_{\Omega_{n}}\left|\widetilde{w_{n}}(t)\right|^{2}\left(a \partial_{t} \Phi_{\beta, \varepsilon}-\Delta \Phi_{\beta, \varepsilon}\right) d x
\end{aligned}
$$

where we have put $\widetilde{w_{n}}(t)=w_{n}(t) \Phi_{\beta, \varepsilon}^{-1}$. By using the property of $\Phi_{\beta, \varepsilon}$ as a supersolution in Lemma 3.7, we deduce

$$
\int_{\Omega_{n}}\left|w_{n}(t)\right|^{2} \Phi_{\beta, \varepsilon}(\cdot, t, 1)^{-1} d \mu \leq \int_{\Omega_{n}}|f|^{2} \Phi_{\beta, \varepsilon}(\cdot, 0,1)^{-1} d \mu
$$

and therefore we have

$$
(1+t)^{\beta} \int_{\Omega_{n}}\left|w_{n}(t)\right|^{2} d \mu \leq C \int_{\Omega_{n}}|f|^{2}\langle x\rangle^{(2-\alpha) \beta} d \mu \leq C \int_{\Omega}|f|^{2}\langle x\rangle^{(2-\alpha) \beta} d \mu .
$$

Then we prove $T_{n}(t) f \rightarrow T(t) f$ in $L_{d \mu}^{2}$ by employing Trotter-Kato convergence theorem (see EngelNagel [4, Theorem 4.8]). For $f \in L_{d \mu}^{2}$, set $u_{n}=\left(1-L_{n}\right)^{-1} f$. Since the semigroups $\left\{T_{n}(t)\right\}_{t \geq 0}$ are positive, we may assume $f \geq 0$ and $u_{n} \geq 0$ without loss of generality. In view of maximum principle, the restriction $\chi_{n} u_{n}$ is nondecreasing with respect to $n$, where $\chi_{n}$ is the indicator function on $\Omega_{n}$. Moreover, observing that

$$
\int_{\Omega_{n}} u_{n} \varphi d \mu+\int_{\Omega_{n}} \nabla u_{n} \cdot \nabla \varphi d x=\int_{\Omega_{n}} f \varphi d \mu, \quad \varphi \in H_{0}^{1}\left(\Omega_{n}\right)
$$

we see from the choice $\varphi=u_{n}$ that $\left\|u_{n}\right\|_{L_{d \mu}^{2}\left(\Omega_{n}\right)} \leq\|f\|_{L_{d \mu}^{2}}$ and $\left\|\nabla u_{n}\right\|_{L^{2}\left(\Omega_{n}\right)} \leq\|f\|_{L_{d \mu}^{2}}$. This yields that there exists $u \in L_{d \mu}^{2} \cap \dot{H}^{1}(\Omega)$ such that $\chi_{n} u_{n} \rightarrow u$ in $L_{d \mu}^{2}$ with $\|\nabla u\|_{L^{2}(\Omega)} \leq\|f\|_{L_{d \mu}^{2}}$. Since $u$ satisfies the Dirichlet boundary condition on $\partial \Omega$, $u$ belongs to $D(\mathfrak{a})$. Letting $n \rightarrow \infty$ in (4.4), we have

$$
\int_{\Omega} u \varphi d \mu+\int_{\Omega} \nabla u \cdot \nabla \varphi d x=\int_{\Omega} f \varphi d \mu, \quad \varphi \in C_{c}^{\infty}(\Omega) .
$$

Therefore $u \in D(L)$ and $u-L u=f$. This gives $\left(I-L_{n}\right)^{-1} f \rightarrow(I-L)^{-1} f$ in $L_{d \mu}^{2}$ as $n \rightarrow \infty$. The Trotter-Kato theorem implies $T_{n}(t) f \rightarrow T(t) f$ in $L_{d \mu}^{2}$ as $n \rightarrow \infty$. Consequently, (4.3) implies the desired estimate. The proof is complete. 


\section{Application to weighted energy estimates for damped wave equations}

In this section, we discuss the asymptotic behavior of the solution to the initial-boundary value problem of the damped wave equation

$$
\begin{cases}\partial_{t}^{2} u(x, t)-\Delta u(x, t)+a(x) \partial_{t} u(x, t)=0, & (x, t) \in \Omega \times(0, \infty), \\ u(x, t)=0, & (x, t) \in \partial \Omega \times(0, \infty), \\ u(0, x)=u_{0}(x), \quad \partial_{t} u(0, x)=u_{1}(x), & x \in \Omega .\end{cases}
$$

Here, $\Omega$ is an exterior domain in $\mathbb{R}^{N}$ with $N \geq 2$, namely, $\mathbb{R}^{N} \backslash \Omega$ is compact. We assume that the boundary $\partial \Omega$ is smooth. We can also treat the case $\Omega=\mathbb{R}^{N}$ with $N \geq 1$. In that case, we omit the boundary condition from (5.1).

We assume that the coefficient of the damping term $a(x)$ is a smooth positive function defined on $\mathbb{R}^{n}$ and satisfying

$$
\lim _{|x| \rightarrow \infty}\left(|x|^{\alpha} a(x)\right)=a_{0}
$$

with some $\alpha \in[0,1)$ and $a_{0}>0$. The initial data satisfy $\left(u_{0}, u_{1}\right) \in\left(H^{2}(\Omega) \cap H_{0}^{1}(\Omega)\right) \times H_{0}^{1}(\Omega)$.

It is known that (5.1) has a unique solution

$$
u \in C^{2}\left([0, \infty) ; L^{2}(\Omega)\right) \cap C^{1}\left([0, \infty) ; H_{0}^{1}(\Omega)\right) \cap C\left([0, \infty) ; H^{2}(\Omega)\right)
$$

(see [7, Theorem 2]).

In view of the validity of weighted Hardy inequality

$$
\int_{\Omega}\langle x\rangle^{2(\sigma-1)}|u(x)|^{2} \leq C \int_{\Omega}\langle x\rangle^{2 \sigma}|\nabla u(x)|^{2} d x
$$

which crucially affects to the validity of Lemma 5.2, we will split the proofs of weighted energy estimates for multi-dimensional case and one-dimensional case.

\subsection{Weighted energy estimates for $N \geq 2$}

Let $t_{0} \geq 1$ be sufficiently large determined later and let

$$
\Psi\left(x, t ; t_{0}\right):=t_{0}+t+A_{\varepsilon}(x),
$$

where the function $A_{\varepsilon}(x)$ is given in Lemma 3.2. We first show the relation of $\Phi_{\beta, \varepsilon}\left(x, t ; t_{0}\right)$ and $\Psi\left(x, t ; t_{0}\right)$. Lemma 5.1. Let $\Phi_{\beta, \varepsilon}\left(x, t ; t_{0}\right)$ and $\Psi\left(x, t ; t_{0}\right)$ be defined in Definition 3.6 and (5.4), respectively. Then the followings are hold.

(i) If $\beta \geq 0$, then there exists a constant $C_{\alpha, \beta, \varepsilon}>0$ such that

$$
\left|\Phi_{\beta, \varepsilon}\left(x, t ; t_{0}\right)\right| \leq C_{\alpha, \beta, \varepsilon} \Psi\left(x, t ; t_{0}\right)^{-\beta}
$$

for any $(x, t) \in \mathbb{R}^{N} \times[0, \infty)$. 
(ii) If $\beta \in\left(0, \gamma_{\varepsilon}\right)$, then there exists a constant $c_{\alpha, \beta, \varepsilon}>0$ such that

$$
\left|\Phi_{\beta, \varepsilon}\left(x, t ; t_{0}\right)\right| \geq c_{\alpha, \beta, \varepsilon} \Psi\left(x, t ; t_{0}\right)^{-\beta}
$$

for any $(x, t) \in \mathbb{R}^{N} \times[0, \infty)$.

This lemma directly follows from Lemma 3.5 and we omit the detail.

Next, we prepare a Hardy-type inequality with the weight function $\Psi$.

Lemma 5.2 (Hardy-type inequality). For every $w \in H_{0}^{1}(\Omega)$ having a compact support on $\mathbb{R}^{N}$ and $\lambda>$ $-\frac{N-2+2 \varepsilon(N-\alpha)}{2-\alpha+\varepsilon(N-\alpha)}$, there exists a positive constant $C=C_{N, \alpha, \varepsilon, \lambda}$ such that

$$
\int_{\Omega}|w|^{2} a(x) \Psi^{\lambda-1} d x \leq C \int_{\Omega}|\nabla w|^{2} \Psi^{\lambda} d x
$$

Remark 5.1. (i) The constant $C_{N, \alpha, \varepsilon, \lambda}$ in the above lemma is explicitly given by

$$
C_{N, \alpha, \varepsilon, \lambda}=4\left(\frac{2-\alpha}{N-\alpha}+\varepsilon\right) \min \left\{1-\varepsilon, 1-\varepsilon+(\lambda-1)\left(\frac{2-\alpha}{N-\alpha}+\varepsilon\right)\right\}^{-2} .
$$

(ii) Lemma 5.2 holds even when $\Omega=\mathbb{R}^{N}$ with $N=1$. However, due to the restriction on $\lambda$, we cannot apply it to weighted energy estimates. This is the difference with one-dimensional case.

Proof of Lemma 5.2. The proof is similar to that of [24, Lemma 3.5]. First, noting $\nabla \Psi=\nabla A_{\varepsilon}$ and $\Delta \Psi=\Delta A_{\varepsilon}$, and using Lemma 3.2, we calculate

$$
\begin{aligned}
\operatorname{div}\left(\Psi^{\lambda-1} \nabla \Psi\right) & =(\Delta \Psi) \Psi^{\lambda-1}+(\lambda-1)|\nabla \Psi|^{2} \Psi^{\lambda-2} \\
& \geq(1-\varepsilon) a(x) \Psi^{\lambda-1}+(\lambda-1)\left|\nabla A_{\varepsilon}(x)\right|^{2} \Psi^{\lambda-2} \\
& =\left[(1-\varepsilon)\left(t_{0}+t+A_{\varepsilon}(x)\right)+(\lambda-1) \frac{\left|\nabla A_{\varepsilon}(x)\right|^{2}}{a(x)}\right] a(x) \Psi^{\lambda-2} \\
& =\left[(1-\varepsilon)\left(t_{0}+t\right)+\left(1-\varepsilon+(\lambda-1) \frac{\left|\nabla A_{\varepsilon}(x)\right|^{2}}{a(x) A_{\varepsilon}(x)}\right) A_{\varepsilon}(x)\right] a(x) \Psi^{\lambda-2} \\
& \geq \min \left\{1-\varepsilon, 1-\varepsilon+(\lambda-1)\left(\frac{2-\alpha}{N-\alpha}+\varepsilon\right)\right\} a(x) \Psi^{\lambda-1}
\end{aligned}
$$

Since $\varepsilon \in(0,1)$ and $\lambda>-\frac{N-2+2 \varepsilon(N-\alpha)}{2-\alpha+\varepsilon(N-\alpha)}$, all members in the minimum are positive. On the one hand, the integration by parts and the Schwarz inequality lead to

$$
\begin{aligned}
\int_{\Omega}|w|^{2} \operatorname{div}\left(\Psi^{\lambda-1} \nabla \Psi\right) d x & =-2 \int_{\Omega} w(\nabla w \cdot \nabla \Psi) \Psi^{\lambda-1} d x \\
& \leq 2\left(\int_{\Omega}|w|^{2} a(x) \Psi^{\lambda-1} d x\right)^{1 / 2}\left(\int_{\Omega}|\nabla w|^{2} \frac{|\nabla \Psi|^{2}}{a(x)} \Psi^{\lambda-1} d x\right)^{1 / 2} \\
& \leq 2\left(\int_{\Omega}|w|^{2} a(x) \Psi^{\lambda-1} d x\right)^{1 / 2}\left(\left(\frac{2-\alpha}{N-\alpha}+\varepsilon\right) \int_{\Omega}|\nabla w|^{2} \Psi^{\lambda} d x\right)^{1 / 2}
\end{aligned}
$$

Here we have used that

$$
\frac{|\nabla \Psi|^{2}}{a(x)}=\frac{|\nabla A(x)|^{2}}{a(x) A(x)} A(x) \leq\left(\frac{2-\alpha}{N-\alpha}+\varepsilon\right) \Psi\left(x, t ; t_{0}\right)
$$

holds by Lemma 3.2. Putting all the estimates together, we obtain the desired assertion. 
Lemma 5.3 ([20] Lemma 2.5). For $\Phi \in C^{2}(\bar{\Omega}), u \in H^{2}(\Omega) \cap H_{0}^{1}(\Omega)$ and $\delta \in(0,1 / 2)$, we have

$$
\int_{\Omega} u \Delta u \Phi^{-1+2 \delta} d x \leq-\frac{\delta}{1-\delta} \int_{\Omega}|\nabla u|^{2} \Phi^{-1+2 \delta} d x+\frac{1-2 \delta}{2} \int_{\Omega} u^{2}(\Delta \Phi) \Phi^{-2+2 \delta} d x .
$$

The proof in done by integration by parts and can be found in [20, Lemma 2.5] and we omit the detail.

Definition 5.4 (Weighted energy). Let $\delta \in(0,1 / 2), \varepsilon \in(0,1), \lambda \in\left(0,(1-2 \delta) \gamma_{\varepsilon}\right)$ and $\beta=\lambda /(1-2 \delta)$. Let $t_{0} \geq 1$ and $v>0$ be sufficiently large and small, respectively, and determined later. We define the weighted energy

$$
\begin{aligned}
E_{1}\left(t ; t_{0}\right) & :=\int_{\Omega}\left(|\nabla u(x, t)|^{2}+\left|\partial_{t} u(x, t)\right|^{2}\right) \Psi\left(x, t ; t_{0}\right)^{\lambda+\frac{\alpha}{2-\alpha}} d x, \\
E_{0}\left(t ; t_{0}\right) & :=\int_{\Omega}\left(2 u(x, t) \partial_{t} u(x, t)+a(x)|u(x, t)|^{2}\right) \Phi_{\beta, \varepsilon}\left(x, t ; t_{0}\right)^{-1+2 \delta} d x, \\
E\left(t ; t_{0}, v\right) & :=E_{1}\left(t ; t_{0}\right)+v E_{0}\left(t ; t_{0}\right), \\
\widetilde{E}_{1}\left(t ; t_{0}\right) & :=\left(t_{0}+t\right) \int_{\Omega}\left(|\nabla u(x, t)|^{2}+\left|\partial_{t} u(x, t)\right|^{2}\right) \Psi\left(x, t ; t_{0}\right)^{\lambda} d x
\end{aligned}
$$

for $t \geq 0$.

Our strategy of the weighted energy estimates is the following: First, combining the estimates for $E_{1}\left(t ; t_{0}\right)$ and $E_{0}\left(t ; t_{0}\right)$, we give an energy estimate for $E\left(t ; t_{0}, v\right)$. Then, using it, we derive the boundedness of $\widetilde{E}_{1}\left(t ; t_{0}\right)$, which gives a sharper decay estimate for $\left(\nabla u, \partial_{t} u\right)$. The main result of this subsection is the following:

Theorem 5.5. Assume (5.2). Then there exist $t_{*} \geq 1$ and $v>0$ such that for any $t_{0} \geq t_{*}$ the following holds: suppose that the initial data satisfy

$$
I_{0}:=\int_{\Omega}\left(\left|\nabla u_{0}(x)\right|^{2}+\left|u_{1}(x)\right|^{2}\right) \Psi\left(x, 0 ; t_{0}\right)^{\lambda+\frac{\alpha}{2-\alpha}} d x+\int_{\Omega} a(x)\left|u_{0}(x)\right|^{2} \Psi\left(x, 0 ; t_{0}\right)^{\lambda} d x<\infty .
$$

Let $u$ be the solution of (5.1) in the class (5.3). Then, we have

$$
\begin{aligned}
& E_{1}\left(t ; t_{0}\right)+\widetilde{E}_{1}\left(t ; t_{0}\right)+\int_{\Omega} a(x)|u(x, t)|^{2} \Psi\left(x, t ; t_{0}\right)^{\lambda} d x \\
& \quad+\int_{0}^{t} \int_{\Omega}|\nabla u(x, \tau)|^{2} \Psi\left(x, \tau ; t_{0}\right)^{\lambda} d x d \tau \\
& \quad+\int_{0}^{t} \int_{\Omega} a(x)\left|\partial_{t} u(x, \tau)\right|^{2} \Psi\left(x, \tau ; t_{0}\right)^{\lambda}\left[\left(t_{0}+\tau\right)+\Psi\left(x, \tau ; t_{0}\right)^{\frac{\alpha}{2-\alpha}}\right] d x d \tau \\
& \leq C I_{0}
\end{aligned}
$$

for $t \geq 0$ with some constant $C=C\left(N, \alpha, \delta, \varepsilon, \lambda, t_{0}, v\right)>0$.

\subsubsection{Proof of Theorem 5.5}

In the proof of weighted energy estimates, we will assume that the initial data $\left(u_{0}, u_{1}\right)$ (and also the solution $u$ by finite propagation property) are compactly supported. All estimates proved below can be extended to the case of non-compactly supported initial data via an approximation with a cut-off procedure.

We split the proof of Theorem 5.5 into the following four lemmas. 
Lemma 5.6. Under the assumption on Theorem 5.5, there exists a constant $t_{1} \geq 1$ such that for any $t_{0} \geq t_{1}$ and $t \geq 0$, we have

$$
\frac{d}{d t} E_{1}\left(t ; t_{0}\right) \leq-\int_{\Omega} a(x)\left|\partial_{t} u(x, t)\right|^{2} \Psi\left(x, t ; t_{0}\right)^{\lambda+\frac{\alpha}{2-\alpha}} d x+C \int_{\Omega}|\nabla u(x, t)|^{2} \Psi\left(x, t ; t_{0}\right)^{\lambda+\frac{\alpha}{2-\alpha}-1} d x
$$

with some constant $C=C\left(N, \alpha, \varepsilon, \lambda, t_{0}\right)>0$.

Proof. Since $u$ is a solution of (5.1), we compute

$$
\begin{aligned}
\frac{d}{d t} E_{1}\left(t ; t_{0}\right)= & 2 \int_{\Omega}\left(\nabla \partial_{t} u \cdot \nabla u+\partial_{t} u \partial_{t}^{2} u\right) \Psi^{\lambda+\frac{\alpha}{2-\alpha}} d x \\
& +\left(\lambda+\frac{\alpha}{2-\alpha}\right) \int_{\Omega}\left(|\nabla u|^{2}+\left|\partial_{t} u\right|^{2}\right) \Psi^{\lambda+\frac{\alpha}{2-\alpha}-1} d x \\
= & -2 \int_{\Omega} a(x)\left|\partial_{t} u\right|^{2} \Psi^{\lambda+\frac{\alpha}{2-\alpha}} d x-2\left(\lambda+\frac{\alpha}{2-\alpha}\right) \int_{\Omega} \partial_{t} u(\nabla u \cdot \nabla \Psi) \Psi^{\lambda+\frac{\alpha}{2-\alpha}-1} d x \\
& +\left(\lambda+\frac{\alpha}{2-\alpha}\right) \int_{\Omega}\left(|\nabla u|^{2}+\left|\partial_{t} u\right|^{2}\right) \Psi^{\lambda+\frac{\alpha}{2-\alpha}-1} d x
\end{aligned}
$$

By the Schwarz inequality

$$
\left|-2\left(\lambda+\frac{\alpha}{2-\alpha}\right) \partial_{t} u(\nabla u \cdot \nabla \Psi)\right| \leq \frac{a(x)}{2}\left|\partial_{t} u\right|^{2} \Psi+C|\nabla u|^{2} \frac{|\nabla \Psi|^{2}}{a(x) \Psi}
$$

and noting

$$
\frac{|\nabla \Psi|^{2}}{a(x) \Psi} \leq \frac{\left|\nabla A_{\varepsilon}(x)\right|^{2}}{a(x) A_{\varepsilon}(x)} \leq \frac{2-\alpha}{N-\alpha}+\varepsilon,
$$

which follows from (3.9), we conclude

$$
\frac{d}{d t} E_{1}\left(t ; t_{0}\right) \leq \int_{\Omega}\left|\partial_{t} u\right|^{2}\left(-2 a(x)+\frac{a(x)}{2}+\left(\lambda+\frac{\alpha}{2-\alpha}\right) \Psi^{-1}\right) \Psi^{\lambda+\frac{\alpha}{2-\alpha}} d x+C \int_{\Omega}|\nabla u|^{2} \Psi^{\lambda+\frac{\alpha}{2-\alpha}-1} d x .
$$

Finally, by $\Psi^{-1} \leq t_{0}^{-1+\frac{\alpha}{2-\alpha}} A(x)^{-\frac{\alpha}{2-\alpha}} \leq C t_{0}^{-1+\frac{\alpha}{2-\alpha}} a(x)$, taking $t_{1} \geq 1$ sufficiently large, we have the desired estimate.

Lemma 5.7. Under the assumption on Theorem 5.5, there exists a constant $t_{2} \geq 1$ such that for any $t_{0} \geq t_{2}$ and $t \geq 0$, we have

$$
\frac{d}{d t} E_{0}\left(t ; t_{0}\right) \leq-\eta_{0} \int_{\Omega}|\nabla u(x, t)|^{2} \Psi\left(x, t ; t_{0}\right)^{\lambda} d x+C \int_{\Omega}\left|\partial_{t} u(x, t)\right|^{2} \Psi\left(x, t ; t_{0}\right)^{\lambda} d x
$$

with some constants $\eta_{0}=\eta_{0}\left(N, \alpha, \varepsilon, \delta, \lambda, t_{0}\right)>0$ and $C=C\left(N, \alpha, \varepsilon, \delta, \lambda, t_{0}\right)>0$.

Proof. Since $u$ is a solution of (5.1), we compute

$$
\begin{aligned}
\frac{d}{d t} E_{0}\left(t ; t_{0}\right)= & 2 \int_{\Omega}\left|\partial_{t} u\right|^{2} \Phi_{\beta, \varepsilon}^{-1+2 \delta} d x+2 \int_{\Omega} u\left(\partial_{t}^{2} u+a(x) \partial_{t} u\right) \Phi_{\beta, \varepsilon}^{-1+2 \delta} d x \\
& -(1-2 \delta) \int_{\Omega}\left(2 u \partial_{t} u+a(x)|u|^{2}\right) \Phi_{\beta, \varepsilon}^{-2+2 \delta} \partial_{t} \Phi_{\beta, \varepsilon} d x \\
= & 2 \int_{\Omega}\left|\partial_{t} u\right|^{2} \Phi_{\beta, \varepsilon}^{-1+2 \delta} d x+2 \int_{\Omega} u \Delta u \Phi_{\beta, \varepsilon}^{-1+2 \delta} d x \\
& -2(1-2 \delta) \int_{\Omega} u \partial_{t} u \Phi_{\beta, \varepsilon}^{-2+2 \delta} \partial_{t} \Phi_{\beta, \varepsilon} d x-(1-2 \delta) \int_{\Omega} a(x)|u|^{2} \Phi_{\beta, \varepsilon}^{-2+2 \delta} \partial_{t} \Phi_{\beta, \varepsilon} d x .
\end{aligned}
$$


Lemma 5.3 with $\Phi=\Phi_{\beta, \varepsilon}$ yields

$$
\begin{aligned}
\frac{d}{d t} E_{0}\left(t ; t_{0}\right) \leq & 2 \int_{\Omega}\left|\partial_{t} u\right|^{2} \Phi_{\beta, \varepsilon}^{-1+2 \delta} d x-\frac{2 \delta}{1-\delta} \int_{\Omega}|\nabla u|^{2} \Phi_{\beta, \varepsilon}^{-1+2 \delta} d x \\
& -2(1-2 \delta) \int_{\Omega} u \partial_{t} u \Phi_{\beta, \varepsilon}^{-2+2 \delta} \partial_{t} \Phi_{\beta, \varepsilon} d x-(1-2 \delta) \int_{\Omega}|u|^{2} \Phi_{\beta, \varepsilon}^{-2+2 \delta}\left(a(x) \partial_{t} \Phi_{\beta, \varepsilon}-\Delta \Phi_{\beta, \varepsilon}\right) d x .
\end{aligned}
$$

By Lemma 3.7, $\Phi_{\beta, \varepsilon}$ satisfies

$$
a(x) \partial_{t} \Phi_{\beta, \varepsilon}-\Delta \Phi_{\beta, \varepsilon} \geq 0 .
$$

Moreover, by noting $\beta \in\left(0, \gamma_{\varepsilon}\right)$, we apply Lemmas 5.1 and 3.8 to obtain

$$
\begin{aligned}
\frac{d}{d t} E_{0}\left(t ; t_{0}\right) \leq & \frac{2}{c_{\alpha, \beta, \varepsilon}} \int_{\Omega}\left|\partial_{t} u\right|^{2} \Psi^{\lambda} d x-\frac{2 \delta}{(1-\delta) C_{\alpha, \beta, \varepsilon}} \int_{\Omega}|\nabla u|^{2} \Psi^{\lambda} d x \\
& +\frac{2(1-2 \delta) \beta C_{\alpha, \beta+1, \varepsilon}}{c_{\alpha, \beta, \varepsilon}^{2-2 \delta}} \int_{\Omega}\left|u \| \partial_{t} u\right| \Psi^{\lambda-1} d x
\end{aligned}
$$

With the aid of Lemma 5.2, the last term is estimated as

$$
\begin{aligned}
\int_{\Omega}|u|\left|\partial_{t} u\right| \Psi^{\lambda-1} d x & \leq\left(\int_{\Omega} a(x)|u|^{2} \Psi^{\lambda-1} d x\right)^{1 / 2}\left(\int_{\Omega} a(x)^{-1}\left|\partial_{t} u\right|^{2} \Psi^{\lambda-1} d x\right)^{1 / 2} \\
& \leq C\left(\int_{\Omega}|\nabla u|^{2} \Psi^{\lambda} d x\right)^{1 / 2}\left(\int_{\Omega} a(x)^{-1}\left|\partial_{t} u\right|^{2} \Psi^{\lambda-1} d x\right)^{1 / 2} \\
& \leq C\left(t+t_{0}\right)^{-\frac{1-\alpha}{2-\alpha}}\left(\int_{\Omega}|\nabla u|^{2} \Psi^{\lambda} d x\right)^{1 / 2}\left(\int_{\Omega}\left|\partial_{t} u\right|^{2} \Psi^{\lambda} d x\right)^{1 / 2} \\
& \leq C \int_{\Omega}\left|\partial_{t} u\right|^{2} \Psi^{\lambda} d x+C t_{0}^{-\frac{2(1-\alpha)}{2-\alpha}} \int_{\Omega}|\nabla u|^{2} \Psi^{\lambda} d x
\end{aligned}
$$

Here, for the third inequality step we have used the following:

$$
\frac{1}{a(x) \Psi\left(x, t ; t_{0}\right)} \leq C \frac{\langle x\rangle^{\alpha}}{t+t_{0}+A_{\varepsilon}(x)} \leq C \frac{1}{\left(t+t_{0}\right)^{\frac{2(1-\alpha)}{2-\alpha}}} \frac{\langle x\rangle^{\alpha}}{A_{\varepsilon}(x)^{\frac{\alpha}{2-\alpha}}} \leq C \frac{1}{\left(t+t_{0}\right)^{\frac{2(1-\alpha)}{2-\alpha}}} .
$$

Thus, taking $t_{2} \geq 1$ sufficiently large, we conclude

$$
\frac{d}{d t} E_{0}\left(t ; t_{0}\right) \leq-\eta_{0} \int_{\Omega}|\nabla u|^{2} \Psi^{\lambda} d x+C \int_{\Omega}\left|\partial_{t} u\right|^{2} \Psi^{\lambda} d x
$$

for $t_{0} \geq t_{2}$ and $t \geq 0$ with some constant $\eta_{0}>0$.

Noting $\Psi^{\lambda} \leq C a(x) \Psi^{\lambda+\frac{\alpha}{2-\alpha}}$ and combining Lemmas 5.6 and 5.7, we have an energy estimate for $E\left(t ; t_{0}, v\right)$.

Lemma 5.8. Under the assumption on 5.5, for any $t_{0} \geq \max \left\{t_{1}, t_{2}\right\}$, there exists $v=v\left(N, \alpha, \varepsilon, \delta, \lambda, t_{0}\right)>0$ such that

$$
E\left(t ; t_{0}, v\right) \geq c \int_{\Omega}\left(|\nabla u(x, t)|^{2}+\left|\partial_{t} u(x, t)\right|^{2}\right) \Psi\left(x, t ; t_{0}\right)^{\lambda+\frac{\alpha}{2-\alpha}} d x+c \int_{\Omega} a(x)|u(x, t)|^{2} \Psi\left(x, t ; t_{0}\right)^{\lambda} d x
$$


and

$$
\begin{aligned}
& E\left(t ; t_{0}, v\right)+\int_{0}^{t} \int_{\Omega}\left(|\nabla u(x, \tau)|^{2}+\left|\partial_{t} u(x, \tau)\right|^{2}\right) \Psi\left(x, \tau ; t_{0}\right)^{\lambda} d x d \tau \\
& \quad+\int_{0}^{t} \int_{\Omega} a(x)\left|\partial_{t} u(x, \tau)\right|^{2} \Psi\left(x, \tau ; t_{0}\right)^{\lambda+\frac{\alpha}{2-\alpha}} d x d \tau \\
& \leq C E\left(0 ; t_{0}, v\right)
\end{aligned}
$$

hold for $t \geq 0$ with some constants $c=c\left(N, \alpha, \varepsilon, \delta, \lambda, t_{0}\right)>0$ and $C=C\left(N, \alpha, \varepsilon, \delta, \lambda, t_{0}\right)>0$.

Finally, using Lemma 5.8, we give the following energy estimate for $\widetilde{E}_{1}\left(t ; t_{0}\right)$.

Lemma 5.9. Under the assumption on Theorem 5.5, for any $t_{0} \geq \max \left\{t_{1}, t_{2}\right\}$, there exists $v=v\left(N, \alpha, \varepsilon, \delta, \lambda, t_{0}\right)>$ 0 such that

$$
\widetilde{E}_{1}\left(t ; t_{0}\right)+\int_{0}^{t}\left(t_{0}+\tau\right) \int_{\Omega} a(x)\left|\partial_{t} u(x, \tau)\right|^{2} \Psi\left(x, \tau ; t_{0}\right)^{\lambda} d x d \tau \leq C E\left(0 ; t_{0}\right)
$$

with some constant $C=C\left(N, \alpha, \varepsilon, \delta, \lambda, t_{0}\right)>0$.

Proof. By integration by parts and the Schwarz inequality, we compute

$$
\begin{aligned}
\frac{d}{d t} \widetilde{E}_{1}\left(t ; t_{0}\right)= & \int_{\Omega}\left(|\nabla u|^{2}+\left|\partial_{t} u\right|^{2}\right)\left[\Psi^{\lambda}+\lambda\left(t_{0}+t\right) \Psi^{\lambda-1}\right] d x \\
& +2\left(t_{0}+t\right) \int_{\Omega}\left(\nabla \partial_{t} u \cdot \nabla u+\partial_{t} u \partial_{t}^{2} u\right) \Psi^{\lambda} d x \\
\leq & (\lambda+1) \int_{\Omega}\left(|\nabla u|^{2}+\left|\partial_{t} u\right|^{2}\right) \Psi^{\lambda} d x \\
& -2\left(t_{0}+t\right) \int_{\Omega} a(x)\left|\partial_{t} u\right|^{2} \Psi^{\lambda} d x-2 \lambda\left(t_{0}+t\right) \int_{\Omega} \partial_{t} u(\nabla u \cdot \nabla \Psi) \Psi^{\lambda-1} d x \\
\leq & (\lambda+1) \int_{\Omega}\left(|\nabla u|^{2}+\left|\partial_{t} u\right|^{2}\right) \Psi^{\lambda} d x \\
& -\left(t_{0}+t\right) \int_{\Omega} a(x)\left|\partial_{t} u\right|^{2} \Psi^{\lambda} d x+\lambda^{2}\left(t_{0}+t\right) \int_{\Omega}|\nabla u|^{2} \frac{|\nabla \Psi|^{2}}{a(x) \Psi} \Psi^{\lambda-1} d x \\
\leq & C \int_{\Omega}\left(|\nabla u|^{2}+\left|\partial_{t} u\right|^{2}\right) \Psi^{\lambda} d x-\left(t_{0}+t\right) \int_{\Omega} a(x)\left|\partial_{t} u\right|^{2} \Psi^{\lambda} d x .
\end{aligned}
$$

Integrating the above on $[0, t]$, applying Lemma 5.8 , and noting $\widetilde{E}_{1}\left(0 ; t_{0}\right) \leq C E\left(0 ; t_{0}, v\right)$, we deduce

$$
\widetilde{E}_{1}\left(t ; t_{0}\right)+\int_{0}^{t}\left(t_{0}+\tau\right) \int_{\Omega} a(x)\left|\partial_{t} u\right|^{2} \Psi^{\lambda} d x \leq C E\left(0 ; t_{0}, v\right),
$$

which completes the proof.

Theorem 5.5 immediately follows from Lemmas 5.6 and 5.9. 


\subsection{Weighted energy estimates for $N=1$}

In the one-dimensional case, instead of Lemma 5.2, we use a modified weight function

$$
\widetilde{\Phi}_{\beta, \varepsilon}\left(x, t ; t_{0}\right):=\left(2-\frac{1}{\left(t_{0}+t\right)^{\frac{2(1-\alpha)}{2-\alpha}}}\right) \Phi_{\beta, \varepsilon}\left(x, t ; t_{0}\right) .
$$

Then, $\widetilde{\Phi}_{\beta, \varepsilon}$ satisfies

$$
a(x) \partial_{t} \widetilde{\Phi}_{\beta, \varepsilon}\left(x, t ; t_{0}\right)-\Delta \widetilde{\Phi}_{\beta, \varepsilon}\left(x, t ; t_{0}\right) \geq \frac{1-\alpha}{2-\alpha} a(x)\left(t_{0}+t\right)^{-2+\frac{\alpha}{2-\alpha}} \widetilde{\Phi}_{\beta, \varepsilon}\left(x, t ; t_{0}\right) .
$$

Using $\widetilde{\Phi}_{\beta, \varepsilon}$, we modify the definition of $E_{0}\left(t ; t_{0}\right)$ as

$$
E_{0}\left(t ; t_{0}\right)=E_{0}\left(t ; t_{0}\right):=\int_{\Omega}\left(2 u(x, t) \partial_{t} u(x, t)+a(x)|u(x, t)|^{2}\right) \widetilde{\Phi}_{\beta, \varepsilon}\left(x, t ; t_{0}\right)^{-1+2 \delta} d x .
$$

Therefore, in the proof of Lemma 5.7, instead of (5.10), we obtain

$$
\begin{aligned}
\frac{d}{d t} E_{0}\left(t ; t_{0}\right) \leq & C \int_{\mathbb{R}}\left|\partial_{t} u\right|^{2} \Psi^{\lambda} d x-\eta \int_{\mathbb{R}}|\nabla u|^{2} \Psi^{\lambda} d x-\eta\left(t_{0}+t\right)^{-2+\frac{\alpha}{2-\alpha}} \int_{\mathbb{R}} a(x)|u|^{2} \Psi^{\lambda} d x \\
& +C \int_{\mathbb{R}}\left|u \| \partial_{t} u\right| \Psi^{\lambda-1} d x
\end{aligned}
$$

with some $C, \eta>0$. The last term is estimated as

$$
\int_{\mathbb{R}}\left|u \| \partial_{t} u\right| \Psi^{\lambda-1} d x \leq \tilde{\eta} \int_{\mathbb{R}}|u|^{2} \Psi^{\lambda-2} d x+C \int_{\mathbb{R}}\left|\partial_{t} u\right|^{2} \Psi^{\lambda} d x .
$$

Noting $\Psi^{-2} \leq\left(t_{0}+t\right)^{-2+\frac{\alpha}{2-\alpha}} A_{\varepsilon}(x)^{-\frac{\alpha}{2-\alpha}} \leq C\left(t_{0}+t\right)^{-2+\frac{\alpha}{2-\alpha}} a(x)$ and taking $\tilde{\eta}$ sufficiently small, we have the same conclusion of Lemma 5.7. The rest part is completely the same as in the case $N \geq 2$, and we have the same conclusion of Theorem 5.5 in the case $N=1$.

\subsection{Weighted energy estimates for higher order derivatives}

In this subsection, we discuss weighted energy estimates for higher order derivatives of the solution. For $k \in \mathbb{N}$, We say that the initial data satisfy the compatibility condition of order $k$ if

$$
u_{\ell}=\Delta u_{\ell-2}-a(x) u_{\ell-1}, \quad\left(u_{\ell-1}, u_{\ell}\right) \in\left(H^{2} \cap H_{0}^{1}(\Omega)\right) \times H_{0}^{1}(\Omega), \quad(\ell=2, \ldots, k+1)
$$

can be successively defined. For $k \in \mathbb{N}$, It is known that if $\left(u_{0}, u_{1}\right) \in\left(H^{k+2} \cap H_{0}^{1}(\Omega)\right) \times\left(H^{k+1} \cap H_{0}^{1}(\Omega)\right)$ fulfill the compatibility condition of order $k$, then the solution of (5.1) satisfies

$$
u \in \bigcap_{\ell=0}^{k+2} C^{\ell}\left([0, \infty) ; H^{k-\ell+2}(\Omega)\right)
$$

in addition to (5.3) (see [7, Theorem 2]). 
Definition 5.10 (Weighted energy of higher order derivatives). Let $k \in \mathbb{N}, \delta \in(0,1 / 2), \varepsilon \in(0,1)$, and $\lambda \in\left(0,(1-2 \delta) \gamma_{\varepsilon}\right)$. Let $t_{0} \geq 1$ and $v_{k, j}>0$ with $j=0,1, \ldots, 2 k$ be sufficiently large and small, respectively, and determined later. For a function $w=w(x, t)$, we define the weighted energy for $t \geq 0$ by

$$
E_{1}^{(k, j)}[w]\left(t ; t_{0}\right):=\left(t_{0}+t\right)^{j} \int_{\Omega}\left(|\nabla w(x, t)|^{2}+\left|\partial_{t} w(x, t)\right|^{2}\right) \Psi\left(x, t ; t_{0}\right)^{\lambda+(2 k+1-j) \frac{\alpha}{2-\alpha}} d x
$$

for $j=0,1, \ldots, 2 k+1$, and

$$
\begin{aligned}
E_{0}^{(k, j)}[w]\left(t ; t_{0}\right) & :=\left(t_{0}+t\right)^{j} \int_{\Omega}\left(2 w(x, t) \partial_{t} w(x, t)+a(x)|w(x, t)|^{2}\right) \Psi\left(x, t ; t_{0}\right)^{\lambda+(2 k-j) \frac{\alpha}{2-\alpha}} d x, \\
E^{(k, j)}[w]\left(t ; t_{0}, v_{k, j}\right) & :=E_{1}^{(k, j)}\left(t ; t_{0}\right)+v_{k, j} E_{0}^{(k, j)}\left(t ; t_{0}\right)
\end{aligned}
$$

for $j=0,1, \ldots, 2 k$.

The main result of this subsection is the following weighted energy estimates for time derivatives of the solution, which improves our previous result in [24, Theorem 4.1].

Theorem 5.11. Let $k \in \mathbb{N}$ and let the initial data $\left(u_{0}, u_{1}\right)$ satisfy the compatibility condition of order $k$. Then, there exist $t_{*} \geq 1$ and $v_{k, j}>0$ with $j=0,1, \ldots, 2 k$ such that for any $t_{0} \geq t_{*}$ the following holds: Assume that the initial data satisfy

$$
I_{k}:=\sum_{\ell=0}^{k}\left[\int_{\Omega}\left(\left|\nabla u_{\ell}(x)\right|^{2}+\left|u_{\ell+1}(x)\right|^{2}\right) \Psi\left(x, 0 ; t_{0}\right)^{\lambda+(2 \ell+1) \frac{\alpha}{2-\alpha}} d x+\int_{\Omega} a(x)\left|u_{\ell}(x)\right|^{2} \Psi\left(x, 0 ; t_{0}\right)^{\lambda+2 \ell \frac{\alpha}{2-\alpha}} d x\right]<\infty .
$$

Let $u$ be the corresponding solution in the class (5.3) and (5.11). Then, we have

$$
\begin{aligned}
& \sum_{j=0}^{2 k+1} E_{1}^{(k, j)}\left[\partial_{t}^{k} u\right]\left(t ; t_{0}\right)+\sum_{j=0}^{2 k}\left(t_{0}+t\right)^{j} \int_{\Omega} a(x)\left|\partial_{t}^{k} u(x, t)\right|^{2} \Psi\left(x, t ; t_{0}\right)^{\lambda+(2 k-j) \frac{\alpha}{2-\alpha}} d x \\
& \quad+\sum_{j=0}^{2 k+1} \int_{0}^{t}\left(t_{0}+\tau\right)^{j} \int_{\Omega} a(x)\left|\partial_{t}^{k+1} u(x, \tau)\right|^{2} \Psi\left(x, \tau ; t_{0}\right)^{\lambda+(2 k+1-j) \frac{\alpha}{2-\alpha}} d x d \tau \\
& \quad+\sum_{j=0}^{2 k} \int_{0}^{t}\left(t_{0}+\tau\right)^{j} \int_{\Omega}\left|\nabla \partial_{t}^{k} u(x, \tau)\right|^{2} \Psi\left(x, \tau ; t_{0}\right)^{\lambda+(2 k-j) \frac{\alpha}{2-\alpha}} d x d \tau \\
& \leq C I_{k}
\end{aligned}
$$

for $t \geq 0$ with some constant $C=C\left(k, N, \alpha, \delta, \varepsilon, \lambda, t_{0}, v_{k, 0}, \ldots, v_{k, 2 k}\right)>0$.

Remark 5.2. If we formally take $k=0$ in the above theorem, then we have the same conclusion of Theorem 5.5. In this sense we interpret that the above theorem is also valid for $k=0$.

\subsubsection{Proof of Theorem 5.11}

We prove Theorem 5.11 by induction. The case $k=0$ has already done by Theorem 5.5 (see Remark 5.2). We assume that Theorem 5.11 is valid for $k-1$.

Next, for the induction step, we prove the following lemma, which shows that if a solution of the damped wave equation (5.1) has a certain space-time bound, then it decays faster than general cases. 
Lemma 5.12. Let $k \in \mathbb{N}$. Let $\left(w_{0}, w_{1}\right)$ satisfy the compatibility condition of order 1 and $w$ be the corresponding solution of (5.1) with the initial data $\left(w_{0}, w_{1}\right)$. Then, there exists $t_{*} \geq 1$ and $v_{k, j}>0$ with $j=0,1, \ldots, 2 k$ such that for any $t_{0} \geq t_{*}$, the following holds: Assume that the initial data satisfy

$$
I=\int_{\Omega}\left(\left|\nabla w_{0}(x)\right|^{2}+\left|w_{1}(x)\right|^{2}\right) \Psi\left(x, 0 ; t_{0}\right)^{\lambda+(2 k+1) \frac{\alpha}{2-\alpha}} d x+\int_{\Omega} a(x)\left|w_{0}(x)\right|^{2} \Psi\left(x, 0 ; t_{0}\right)^{\lambda+2 k \frac{\alpha}{2-\alpha}} d x<\infty
$$

and the solution $w$ satisfies

$$
K=\sum_{j=0}^{2(k-1)+1} \int_{0}^{\infty}\left(t_{0}+\tau\right)^{j} \int_{\Omega} a(x)|w(x, \tau)|^{2} \Psi\left(x, \tau ; t_{0}\right)^{\lambda+(2 k-1+j) \frac{\alpha}{2-\alpha}} d x d \tau<\infty .
$$

Then, we have

$$
\begin{aligned}
& \sum_{j=0}^{2 k+1} E_{1}^{(k, j)}[w]\left(t ; t_{0}\right)+\sum_{j=0}^{2 k}\left(t_{0}+t\right)^{j} \int_{\Omega} a(x)|w(x, t)|^{2} \Psi\left(x, t ; t_{0}\right)^{\lambda+(2 k-j) \frac{\alpha}{2-\alpha}} d x \\
& \quad+\sum_{j=0}^{2 k+1} \int_{0}^{t}\left(t_{0}+\tau\right)^{j} \int_{\Omega} a(x)\left|\partial_{t} w(x, \tau)\right|^{2} \Psi\left(x, \tau ; t_{0}\right)^{\lambda+(2 k+1-j) \frac{\alpha}{2-\alpha}} d x d \tau \\
& \quad+\sum_{j=0}^{2 k} \int_{0}^{t}\left(t_{0}+\tau\right)^{j} \int_{\Omega}|\nabla w(x, \tau)|^{2} \Psi\left(x, \tau ; t_{0}\right)^{\lambda+(2 k-j) \frac{\alpha}{2-\alpha}} d x d \tau \\
& \leq C(I+K)
\end{aligned}
$$

for $t \geq 0$ with some constant $C=C\left(k, N, \alpha, \delta, \varepsilon, \lambda, t_{0}, v_{k, 0}, \ldots, v_{k, 2 k}\right)>0$.

Since the assumption of induction ensures the condition (5.15) when $w=\partial_{t}^{k} u$, we obtain the induction step of the proof of Theorem 5.11 from Lemma 5.12. Therefore, it suffices to show Lemma 5.12 .

The proof of Lemma 5.12 is highly technical. However, the principle is simple, that is, the assumptions of the space-time bound (5.15) and the bound of a certain weighted energy of initial data produce faster energy decay estimates of solutions. Actually, in the first step, by using (5.15), we give an estimate of $E^{(k, 0)}[w]\left(t ; t_{0}\right)$. As a byproduct, we can obtain the boundedness of the third term of (5.16) for $j=0$. Using it, in the second step, we give estimates of $E^{(k, j)}[w]\left(t ; t_{0}\right)$ for $j=1, \ldots, 2 k$ in order, and similarly, we have the boundedness of the third term of (5.16) for $j=1, \ldots, 2 k$ as outgrowths. Finally, by using the $2 k$-th one, in the step 3, we give an estimate of $E_{1}^{(k, 2 k+1)}[w]\left(t ; t_{0}\right)$.

Proof of Lemma 5.12. We divide the proof into the following three steps.

Step 1. An estimate for $E^{(k, 0)}[w]\left(t ; t_{0}, v_{k, 0}\right)$;

Step 2. Estimates of $E^{(k, j)}[w]\left(t ; t_{0}, v_{k, j}\right)$ for $j=1, \ldots, 2 k$;

Step 3. An estimate for $E_{1}^{(k, 2 k+1)}[w]\left(t ; t_{0}\right)$. 
Step 1: An estimate for $E^{(k, 0)}[w]\left(t ; t_{0}, v_{k, 0}\right)$

Lemma 5.13. Under the assumption on Theorem 5.11, there exists a constant $t_{0,1} \geq 1$ such that for any $t_{0} \geq t_{0,1}$ and $t \geq 0$, we have

$\frac{d}{d t} E_{1}^{(k, 0)}[w]\left(t ; t_{0}\right) \leq-\int_{\Omega} a(x)\left|\partial_{t} w(x, t)\right|^{2} \Psi\left(x, t ; t_{0}\right)^{\lambda+(2 k+1) \frac{\alpha}{2-\alpha}} d x+C \int_{\Omega}|\nabla w(x, t)|^{2} \Psi\left(x, t ; t_{0}\right)^{\lambda+(2 k+1) \frac{\alpha}{2-\alpha}-1} d x$ with some constant $C=C\left(k, N, \alpha, \varepsilon, \lambda, t_{0}\right)>0$.

The proof is completely the same as that of Lemma 5.6 and we omit the detail.

Lemma 5.14. Under the assumption on Theorem 5.11, there exists a constant $t_{0,2} \geq 1$ such that for any $t_{0} \geq t_{0,2}$ and $t \geq 0$, we have

$$
\begin{aligned}
\frac{d}{d t} E_{0}^{(k, 0)}[w]\left(t ; t_{0}\right) \leq & -\int_{\Omega}|\nabla w(x, t)|^{2} \Psi\left(x, t ; t_{0}\right)^{\lambda+2 k \frac{\alpha}{2-\alpha}} d x+C \int_{\Omega}\left|\partial_{t} w(x, t)\right|^{2} \Psi\left(x, t ; t_{0}\right)^{\lambda+2 k \frac{\alpha}{2-\alpha}} d x \\
& +C \int_{\Omega} a(x)|w(x, t)|^{2} \Psi\left(x, t ; t_{0}\right)^{\lambda+(2 k-1) \frac{\alpha}{2-\alpha}} d x
\end{aligned}
$$

with some constant $C=C\left(k, N, \alpha, \varepsilon, \lambda, t_{0}\right)>0$.

Proof. By integration by parts, we have

$$
\begin{aligned}
\frac{d}{d t} E_{0}^{(k, 0)}[w]\left(t ; t_{0}\right)= & 2 \int_{\Omega}\left(\left|\partial_{t} w\right|^{2}+w \Delta w\right) \Psi^{\lambda+2 k \frac{\alpha}{2-\alpha}} d x \\
& +\left(\lambda+2 k \frac{\alpha}{2-\alpha}\right) \int_{\Omega}\left(2 w \partial_{t} w+a(x)|w|^{2}\right) \Psi^{\lambda+2 k \frac{\alpha}{2-\alpha}-1} d x \\
= & 2 \int_{\Omega}\left|\partial_{t} w\right|^{2} \Psi^{\lambda+2 k \frac{\alpha}{2-\alpha}} d x-2 \int_{\Omega}|\nabla w|^{2} \Psi^{\lambda+2 k \frac{\alpha}{2-\alpha}} d x \\
& -2\left(\lambda+2 k \frac{\alpha}{2-\alpha}\right) \int_{\Omega} w(\nabla w \cdot \nabla \Psi) \Psi^{\lambda+2 k \frac{\alpha}{2-\alpha}-1} d x \\
& +\left(\lambda+2 k \frac{\alpha}{2-\alpha}\right) \int_{\Omega}\left(2 w \partial_{t} w+a(x)|w|^{2}\right) \Psi^{\lambda+2 k \frac{\alpha}{2-\alpha}-1} d x \\
\leq & C \int_{\Omega}\left|\partial_{t} w\right|^{2} \Psi^{\lambda+2 k \frac{\alpha}{2-\alpha}} d x-\int_{\Omega}|\nabla w|^{2} \Psi^{\lambda+2 k \frac{\alpha}{2-\alpha}} d x \\
& +C \int_{\Omega} a(x)|w|^{2} \Psi^{\lambda+(2 k-1) \frac{\alpha}{2-\alpha}} d x
\end{aligned}
$$

for sufficiently large $t_{0}$. Here we have used the Schwarz inequality

$$
|w(\nabla w \cdot \nabla \Psi)| \leq \eta|\nabla w|^{2} \frac{|\nabla \Psi|^{2}}{a(x) \Psi}+C a(x)|w|^{2} \Psi
$$

with small $\eta>0$ and (5.9). This gives the desired estimate.

Lemma 5.15. Under the assumptions on Lemma 5.12, there exist constants $t_{0, *} \geq \max \left\{t_{0,1}, t_{0,2}\right\}$ and $v_{k, 0}=v_{k, 0}\left(k, N, \alpha, \varepsilon, \lambda, t_{0}\right)>0$ such that for any $t_{0} \geq t_{0, *}$,

$$
\begin{aligned}
E^{(k, 0)}[w]\left(t ; t_{0}\right) \geq & c \int_{\Omega}\left(|\nabla w(x, t)|^{2}+\left|\partial_{t} w(x, t)\right|^{2}\right) \Psi\left(x, t ; t_{0}\right)^{\lambda+(2 k+1) \frac{\alpha}{2-\alpha}} d x \\
& +c \int_{\Omega} a(x)|w(x, t)|^{2} \Psi\left(x, t ; t_{0}\right)^{\lambda+2 k \frac{\alpha}{2-\alpha}} d x
\end{aligned}
$$


and

$$
\begin{aligned}
& E^{(k, 0)}[w]\left(t ; t_{0}\right)+\int_{0}^{t} \int_{\Omega}|\nabla w(x, \tau)|^{2} \Psi\left(x, \tau ; t_{0}\right)^{\lambda+2 k \frac{\alpha}{2-\alpha}} d x d \tau \\
& \quad+\int_{0}^{t} \int_{\Omega} a(x)\left|\partial_{t} w(x, \tau)\right|^{2} \Psi\left(x, \tau ; t_{0}\right)^{\lambda+(2 k+1) \frac{\alpha}{2-\alpha}} d x d \tau \\
& \leq C(I+K)
\end{aligned}
$$

hold for $t \geq 0$ with some constants $c=c\left(k, N, \alpha, \varepsilon, \lambda, t_{0}, v_{k, 0}\right)>0$ and $C=C\left(k, N, \alpha, \varepsilon, \lambda, t_{0}, v_{k, 0}\right)>0$.

Proof. The first assertion is obvious by taking $v_{k, 0}$ sufficiently small. For the second assertion, retaking $v_{k, 0}$ smaller if needed, using Lemmas 5.13 and 5.14 and taking $t_{0, *} \geq \max \left\{t_{0,1}, t_{0,2}\right\}$ sufficiently large, we have

$$
\begin{aligned}
& E^{(k, 0)}[w]\left(t ; t_{0}\right)+\int_{0}^{t} \int_{\Omega}|\nabla w(x, \tau)|^{2} \Psi\left(x, \tau ; t_{0}\right)^{\lambda+2 k \frac{\alpha}{2-\alpha}} d x d \tau \\
& \quad+\int_{0}^{t} \int_{\Omega} a(x)\left|\partial_{t} w(x, \tau)\right|^{2} \Psi\left(x, \tau ; t_{0}\right)^{\lambda+(2 k+1) \frac{\alpha}{2-\alpha}} d x d \tau \\
& \leq C E^{(k, 0)}[w]\left(0 ; t_{0}\right)+C \int_{0}^{t} \int_{\Omega} a(x)|w(x, \tau)|^{2} \Psi\left(x, \tau ; t_{0}\right)^{\lambda+(2 k-1) \frac{\alpha}{2-\alpha}} d x d \tau .
\end{aligned}
$$

The last term is bounded by $C(I+K)$ thanks to the assumption of Lemma 5.12. This leads to the conclusion.

Step 2: Estimates of $E^{(k, j)}[w]\left(t ; t_{0}, v_{k, j}\right)$ for $j=1, \ldots, 2 k$

Next, we estimate $E^{(k, j)}[w]\left(t ; t_{0}, v_{k, j}\right)$ for $j=1, \ldots, 2 k$ in order. The key point is to apply the boundedness of

$$
\int_{0}^{t}\left(t_{0}+\tau\right)^{j-1} \int_{\Omega} a(x)\left(|\nabla w(x, \tau)|^{2}+\left|\partial_{t} x(x, \tau)\right|^{2}\right) \Psi\left(x, \tau ; t_{0}\right)^{\lambda+(2 k+1-j) \frac{\alpha}{2-\alpha}} d x d \tau
$$

obtained in the ( $j-1)$-th step to the estimate in the $j$-th step (see the proof of Lemma 5.18).

Lemma 5.16. Under the assumption on Lemma 5.12, for $j=1, \ldots, 2 k$, there exists a constant $t_{j, 1} \geq 1$ such that for any $t_{0} \geq t_{j, 1}$ and $t \geq 0$, we have

$$
\begin{aligned}
\frac{d}{d t} E_{1}^{(k, j)}[w]\left(t ; t_{0}\right) \leq & -\left(t_{0}+t\right)^{j} \int_{\Omega} a(x)\left|\partial_{t} w(x, t)\right|^{2} \Psi\left(x, t ; t_{0}\right)^{\lambda+(2 k+1-j) \frac{\alpha}{2-\alpha}} d x \\
& +C\left(t_{0}+t\right)^{j-1} \int_{\Omega}\left(|\nabla w(x, t)|^{2}+\left|\partial_{t} w(x, t)\right|^{2}\right) \Psi\left(x, t ; t_{0}\right)^{\lambda+(2 k+1-j) \frac{\alpha}{2-\alpha}} d x
\end{aligned}
$$

with some constant $C=C\left(k, j, N, \alpha, \varepsilon, \lambda, t_{0}\right)>0$. 
Proof. By integration by parts, we have

$$
\begin{aligned}
\frac{d}{d t} E_{1}^{(k, j)}[w]\left(t ; t_{0}\right)= & 2\left(t_{0}+t\right)^{j} \int_{\Omega}\left(\nabla \partial_{t} w \cdot \nabla w+\partial_{t} w \partial_{t}^{2} w\right) \Psi^{\lambda+(2 k+1-j) \frac{\alpha}{2-\alpha}} d x \\
& +\left(t_{0}+t\right)^{j} \int_{\Omega}\left(|\nabla w|^{2}+\left|\partial_{t} w\right|^{2}\right)\left[j\left(t_{0}+t\right)^{-1}+\left(\lambda+(2 k+1-j) \frac{\alpha}{2-\alpha}\right) \Psi^{-1}\right] \Psi^{\lambda+(2 k+1-j) \frac{\alpha}{2-\alpha}} d x \\
\leq & -2\left(t_{0}+t\right)^{j} \int_{\Omega} a(x)\left|\partial_{t} w\right|^{2} \Psi^{\lambda+(2 k+1-j) \frac{\alpha}{2-\alpha}} d x \\
& -2\left(t_{0}+t\right)^{j}\left(\lambda+(2 k+1-j) \frac{\alpha}{2-\alpha}\right) \int_{\Omega} \partial_{t} w(\nabla w \cdot \nabla \Psi) \Psi^{\lambda+(2 k+1-j) \frac{\alpha}{2-\alpha}-1} d x \\
& +C\left(t_{0}+t\right)^{j-1} \int_{\Omega}\left(|\nabla w|^{2}+\left|\partial_{t} w\right|^{2}\right) \Psi^{\lambda+(2 k+1-j) \frac{\alpha}{2-\alpha}} d x \\
\leq & -\left(t_{0}+t\right)^{j} \int_{\Omega} a(x)\left|\partial_{t} w\right|^{2} \Psi^{\lambda+(2 k+1-j) \frac{\alpha}{2-\alpha}} d x \\
& +C\left(t_{0}+t\right)^{j-1} \int_{\Omega}\left(|\nabla w|^{2}+\left|\partial_{t} w\right|^{2}\right) \Psi^{\lambda+(2 k+1-j) \frac{\alpha}{2-\alpha}} d x
\end{aligned}
$$

for sufficiently large $t_{0}$. Here we have used the Schwarz inequality

$$
\left|\partial_{t} w(\nabla w \cdot \nabla \Psi)\right| \leq \eta a(x)\left|\partial_{t} w\right|^{2} \Psi+C|\nabla w|^{2} \frac{|\nabla \Psi|^{2}}{a(x) \Psi}
$$

with small $\eta$ and (5.9). This completes the proof.

Lemma 5.17. Under the assumption on Lemma 5.12 , for $j=1, \ldots, 2 k$, there exists a constant $t_{j, 2} \geq 1$ such that for any $t_{0} \geq t_{j, 2}$ and $t \geq 0$, we have

$$
\begin{aligned}
\frac{d}{d t} E_{0}^{(k, j)}[w]\left(t ; t_{0}\right) \leq & -\left(t_{0}+t\right)^{j} \int_{\Omega}|\nabla w(x, t)|^{2} \Psi\left(x, t ; t_{0}\right)^{\lambda+(2 k-j) \frac{\alpha}{2-\alpha}} d x \\
& +C\left(t_{0}+t\right)^{j} \int_{\Omega}\left|\partial_{t} w(x, t)\right|^{2} \Psi\left(x, t ; t_{0}\right)^{\lambda+(2 k-j) \frac{\alpha}{2-\alpha}} d x \\
& +C\left(t_{0}+t\right)^{j-1} \int_{\Omega} a(x)|w(x, t)|^{2} \Psi\left(x, t ; t_{0}\right)^{\lambda+(2 k-j) \frac{\alpha}{2-\alpha}} d x \\
& +C\left(t_{0}+t\right)^{j-1} \int_{\Omega}\left|\partial_{t} w\right|^{2} \Psi\left(x, t ; t_{0}\right)^{\lambda+(2 k+1-j) \frac{\alpha}{2-\alpha}} d x
\end{aligned}
$$

with some constant $C=C\left(k, j, N, \alpha, \varepsilon, \lambda, t_{0}\right)>0$. 
Proof. By integration by parts, we have

$$
\begin{aligned}
& \frac{d}{d t} E_{0}^{(k, j)}[w]\left(t ; t_{0}\right)=2\left(t_{0}+t\right)^{j} \int_{\Omega}\left(\left|\partial_{t} w\right|^{2}+w \Delta w\right) \Psi^{\lambda+(2 k-j) \frac{\alpha}{2-\alpha}} d x \\
& +\left(t_{0}+t\right)^{j} \int_{\Omega}\left(2 w \partial_{t} w+a(x)|w|^{2}\right)\left[j\left(t_{0}+t\right)^{-1}+\left(\lambda+(2 k-j) \frac{\alpha}{2-\alpha}\right) \Psi^{-1}\right] \Psi^{\lambda+(2 k-j) \frac{\alpha}{2-\alpha}} d x \\
& \leq 2\left(t_{0}+t\right)^{j} \int_{\Omega}\left|\partial_{t} w\right|^{2} \Psi^{\lambda+(2 k-j) \frac{\alpha}{2-\alpha}} d x \\
& -2\left(t_{0}+t\right)^{j} \int_{\Omega}|\nabla w|^{2} \Psi^{\lambda+(2 k-j) \frac{\alpha}{2-\alpha}} d x \\
& -2\left(t_{0}+t\right)^{j}\left(\lambda+(2 k-j) \frac{\alpha}{2-\alpha}\right) \int_{\Omega} w(\nabla w \cdot \nabla \Psi) \Psi^{\lambda+(2 k-j) \frac{\alpha}{2-\alpha}-1} d x \\
& +C\left(t_{0}+t\right)^{j-1} \int_{\Omega}\left(2|w|\left|\partial_{t} w\right|+a(x)|w|^{2}\right) \Psi^{\lambda+(2 k-j) \frac{\alpha}{2-\alpha}} d x \\
& \leq C\left(t_{0}+t\right)^{j} \int_{\Omega}\left|\partial_{t} w\right|^{2} \Psi^{\lambda+(2 k-j) \frac{\alpha}{2-\alpha}} d x \\
& -\left(t_{0}+t\right)^{j} \int_{\Omega}|\nabla w|^{2} \Psi^{\lambda+(2 k-j) \frac{\alpha}{2-\alpha}} d x \\
& +C\left(t_{0}+t\right)^{j-1} \int_{\Omega} a(x)|w|^{2} \Psi^{\lambda+(2 k-j) \frac{\alpha}{2-\alpha}} d x \\
& +C\left(t_{0}+t\right)^{j-1} \int_{\Omega}\left|\partial_{t} w\right|^{2} \Psi^{\lambda+(2 k+1-j) \frac{\alpha}{2-\alpha}} d x .
\end{aligned}
$$

Here, we have used the following inequalities

$$
|w(\nabla w \cdot \nabla \Psi)| \leq \eta|\nabla w|^{2} \frac{|\nabla \Psi|^{2}}{a(x) \Psi}+C a(x)|w|^{2} \Psi, \quad|w|\left|\partial_{t} w\right| \leq a(x)|w|^{2}+C\left|\partial_{t} w\right|^{2} \Psi^{\frac{\alpha}{2-\alpha}}
$$

and (5.9). This completes the proof.

Lemma 5.18. Under the assumptions on Lemma 5.12, for each $j=1, \ldots, 2 k$, there exist constants $t_{j, *} \geq \max \left\{t_{j, 1}, t_{j, 2}\right\}$ and $v_{k, j}=v_{k, j}\left(k, j, N, \alpha, \varepsilon, \lambda, t_{0}\right)>0$ such that for any $t_{0} \geq t_{j, *}$, we have

$$
\begin{aligned}
E^{(k, j)}[w]\left(t ; t_{0}, v_{k, j}\right) \geq & c\left(t_{0}+t\right)^{j} \int_{\Omega}\left(|\nabla w(x, t)|^{2}+\left|\partial_{t} w(x, t)\right|^{2}\right) \Psi\left(x, t ; t_{0}\right)^{\lambda+(2 k+1-j) \frac{\alpha}{2-\alpha}} d x \\
& +c\left(t_{0}+t\right)^{j} \int_{\Omega} a(x)|w(x, t)|^{2} \Psi\left(x, t ; t_{0}\right)^{\lambda+(2 k-j) \frac{\alpha}{2-\alpha}} d x
\end{aligned}
$$

and

$$
\begin{aligned}
& E^{(k, j)}[w]\left(t ; t_{0}, v_{k, j}\right)+\int_{0}^{t}\left(t_{0}+\tau\right)^{j} \int_{\Omega}|\nabla w(x, \tau)|^{2} \Psi\left(x, \tau ; t_{0}\right)^{\lambda+(2 k-j) \frac{\alpha}{2-\alpha}} d x d \tau \\
& \quad+\int_{0}^{t}\left(t_{0}+\tau\right)^{j} \int_{\Omega} a(x)\left|\partial_{t} w(x, \tau)\right|^{2} \Psi\left(x, \tau ; t_{0}\right)^{\lambda+(2 k+1-j) \frac{\alpha}{2-\alpha}} d x d \tau \\
& \leq C(I+K)
\end{aligned}
$$

for $t \geq 0$ with some constants $c=c\left(k, j, N, \alpha, \varepsilon, \lambda, t_{0}, v_{k, j}\right)>0$ and $C=C\left(k, j, N, \alpha, \varepsilon, \lambda, t_{0}, v_{k, 0}, \ldots, v_{k, j}\right)>$ 0 . 
Proof. The first assertion is obvious by taking $v_{k, j}$ sufficiently small. We prove the second assertion for $j=1, \ldots, 2 k$ in order. By Lemmas 5.16 and 5.17, noting that

$$
\left|\partial_{t} w\right|^{2} \Psi\left(x, \tau ; t_{0}\right)^{\lambda+(2 k+1-j) \frac{\alpha}{2-\alpha}} \leq C a\left|\partial_{t} w\right|^{2} \Psi\left(x, \tau ; t_{0}\right)^{\lambda+(2 k+1-(j-1)) \frac{\alpha}{2-\alpha}}
$$

(and retaking $v_{k, j}$ if needed), we have

$$
\begin{aligned}
& E^{(k, j)}[w]\left(t ; t_{0}, v_{k, j}\right)+\int_{0}^{t}\left(t_{0}+\tau\right)^{j} \int_{\Omega}|\nabla w(x, \tau)|^{2} \Psi\left(x, \tau ; t_{0}\right)^{\lambda+(2 k-j) \frac{\alpha}{2-\alpha}} d x d \tau \\
& \quad+\int_{0}^{t}\left(t_{0}+\tau\right)^{j} \int_{\Omega} a(x)\left|\partial_{t} w(x, \tau)\right|^{2} \Psi\left(x, \tau ; t_{0}\right)^{\lambda+(2 k+1-j) \frac{\alpha}{2-\alpha}} d x d \tau \\
& \leq C E^{(k, j)}[w]\left(0 ; t_{0}, v_{k, j}\right)+C \int_{0}^{t}\left(t_{0}+\tau\right)^{j-1} \int_{\Omega} a(x)|w(x, \tau)|^{2} \Psi\left(x, \tau ; t_{0}\right)^{\lambda+(2 k-j) \frac{\alpha}{2-\alpha}} d x \\
& \quad+C \int_{0}^{t}\left(t_{0}+\tau\right)^{j-1} \int_{\Omega}\left(|\nabla w|^{2}+\left|\partial_{t} w\right|^{2}\right) \Psi\left(x, \tau ; t_{0}\right)^{\lambda+(2 k+1-j) \frac{\alpha}{2-\alpha}} d x d \tau
\end{aligned}
$$

We easily see that $E^{(k, j)}[w]\left(0 ; t_{0}, v_{k, j}\right) \leq C I$ with some $C=C\left(k, j, N, \alpha, \varepsilon, \lambda, t_{0}, v_{k, j}\right)>0$. The second term in the right-hand side is bounded by $C K$ thanks to the assumption (5.15). Moreover, the third term in the right-hand side is bounded by $C(I+K)$ because of the assertion for the case $j-1$ (when $j=1$ we apply Lemma 5.15). Continuing this argument from $j=1$ to $j=2 k$, we reach the conclusion.

Step 3: An estimate for $E_{1}^{(k, 2 k+1)}[w]\left(t ; t_{0}\right)$

Finally, we show the boundedness of $E_{1}^{(k, 2 k+1)}[w]\left(t ; t_{0}\right)$, which gives the desired decay for $w$.

Lemma 5.19. Under the assumption on Lemma 5.12, there exists a constant $t_{2 k+1,1} \geq 1$ such that for any $t_{0} \geq t_{2 k+1,1}$ and $t \geq 0$, we have

$$
E_{1}^{(k, 2 k+1)}[w]\left(t ; t_{0}\right)+\int_{0}^{t}\left(t_{0}+\tau\right)^{2 k+1} \int_{\Omega} a(x)\left|\partial_{t} w(x, \tau)\right|^{2} \Psi\left(x, \tau ; t_{0}\right)^{\lambda} d x \leq C(I+K)
$$

with some constant $C=C\left(k, N, \alpha, \varepsilon, \lambda, t_{0}, v_{k, 0}, \ldots, v_{k, 2 k}\right)>0$.

Proof. We first have

$$
\begin{aligned}
\frac{d}{d t} E_{1}^{(k, 2 k+1)[w]\left(t ; t_{0}\right) \leq} & -\left(t_{0}+t\right)^{2 k+1} \int_{\Omega} a(x)\left|\partial_{t} w(x, t)\right|^{2} \Psi\left(x, t ; t_{0}\right)^{\lambda} d x \\
& +C\left(t_{0}+t\right)^{2 k} \int_{\Omega}\left(|\nabla w(x, t)|^{2}+\left|\partial_{t} w(x, t)\right|^{2}\right) \Psi\left(x, t ; t_{0}\right)^{\lambda} d x,
\end{aligned}
$$

which is proved by the same way as Lemma 5.16 and we omit the detail. Integrating the above on $[0, t]$, we have

$$
\begin{aligned}
& E_{1}^{(k, 2 k+1)}[w]\left(t ; t_{0}\right)+\int_{0}^{t}\left(t_{0}+\tau\right)^{2 k+1} \int_{\Omega} a(x)\left|\partial_{t} w\right|^{2} \Psi^{\lambda} d x d \tau \\
& \leq E_{1}^{(k, 2 k+1)}[w]\left(0 ; t_{0}\right)+C \int_{0}^{t}\left(t_{0}+\tau\right)^{2 k} \int_{\Omega}\left(|\nabla w|^{2}+\left|\partial_{t} w\right|^{2}\right) \Psi^{\lambda} d x d \tau .
\end{aligned}
$$

The right-hand side is bounded by $C(I+K)$ thanks to Lemma 5.18 and the inequality $\left|\partial_{t} w\right|^{2} \Psi^{\lambda} \leq$ $C a(x)\left|\partial_{t} w\right|^{2} \Psi^{\lambda+\frac{\alpha}{2-\alpha}}$. The proof is complete.

Finally, combining Lemmas 5.15, 5.18, 5.19, we have the assertion of Lemma 5.12. 


\subsection{Diffusion phenomena}

To close this paper, we finally consider the asymptotic profile of solutions to (5.1). From the viewpoint of weighted energy estimates proved in the previous subsection, we expect that the solution of (5.1) behaves like the one of (4.1) at $t \rightarrow \infty$.

The following is the statement for diffusion phenomena for the problem (5.1). The assertion for the case $a(x) \not \equiv|x|^{-\alpha}$ is an improvement of [24, Theorem 1.2] in which the spatial case $a(x)=|x|^{-\alpha}$ is studied.

Theorem 5.20. Let $\left(u_{0}, u_{1}\right) \in\left(H^{3}(\Omega) \cap H_{0}^{1}(\Omega)\right) \times\left(H^{2}(\Omega) \cap H_{0}^{1}(\Omega)\right)$ satisfies the compatibility condition of order 1 . Let $\delta \in(0,1 / 2), \varepsilon \in(0,1), \lambda \in\left(0,(1-2 \delta) \gamma_{\varepsilon}\right)$ with $\gamma_{\varepsilon}$ defined by (3.12). Assume

$$
I_{1}=\sum_{\ell=0}^{1}\left[\int_{\Omega}\left(\left|\nabla u_{\ell}(x)\right|^{2}+\left|u_{\ell+1}(x)\right|^{2}\right) \Psi\left(x, 0 ; t_{0}\right)^{\lambda+(2 \ell+1) \frac{\alpha}{2-\alpha}} d x+\int_{\Omega} a(x)\left|u_{\ell}(x)\right|^{2} \Psi\left(x, 0 ; t_{0}\right)^{\lambda+2 \ell \frac{\alpha}{2-\alpha}} d x\right]<\infty .
$$

Then, we have the asymptotic estimate

$$
\left\|u(t)-T(t)\left[u_{0}+a^{-1} u_{1}\right]\right\|_{L_{d \mu}^{2}} \leq C(1+t)^{-\lambda / 2} \eta(t) \sqrt{I_{1}}, \quad t \geq 1,
$$

where

$$
\eta(t)= \begin{cases}(1+t)^{-\frac{2(1-\alpha)}{2-\alpha}} \sqrt{\log (2+t)} & \text { if } \lambda \in\left[\frac{2 \alpha}{2-\alpha}, \frac{N-\alpha}{2-\alpha}\right), \\ (1+t)^{-\frac{2(1-\alpha)}{2 \alpha} \lambda} & \text { if } \lambda \in\left(0, \frac{2 \alpha}{2-\alpha}\right) .\end{cases}
$$

Proof. First, by the same argument as [24, Lemma 5.1], we can show that $u(t)$ belongs to $D(L)$ defined in Lemma 4.1 and $a(x)^{-1} \partial_{t}^{2} u \in L^{\infty}\left(0, \infty ; L_{d \mu}^{2}\right)$. Thus, rewriting the equation (5.1) as

$$
\partial_{t} u-a(x)^{-1} \Delta u=-a(x)^{-1} \partial_{t}^{2} u,
$$

and using the semigroup $T(t)$ defined in Lemma 4.1, we have the integral formula

$$
u(t)=T(t) u_{0}-\int_{0}^{t} T(t-s)\left[a^{-1} \partial_{t}^{2} u(s)\right] d s
$$

(see [21, Lemma 4.1] for the detail). Moreover, by integration by parts, we deduce

$$
\begin{aligned}
u(t)= & T(t)\left[u_{0}+a^{-1} u_{1}\right]-\int_{\frac{t}{2}}^{t} T(t-s)\left[a^{-1} \partial_{t}^{2} u(s)\right] d s \\
& -T(t / 2)\left[a^{-1} \partial_{t} u(t / 2)\right]-\int_{0}^{\frac{t}{2}} L T(t-s)\left[a^{-1} \partial_{t} u(s)\right] d s
\end{aligned}
$$

(see [21, p.5715] for the detail). Therefore, we obtain the representation

$$
u(t)-T(t)\left[u_{0}+a^{-1} u_{1}\right]=J_{1}(t)+J_{2}(t)+J_{3}(t),
$$

where

$$
\begin{aligned}
& J_{1}(t)=-\int_{\frac{t}{2}}^{t} T(t-s)\left[a^{-1} \partial_{t}^{2} u(s)\right] d s, \\
& J_{2}(t)=-T(t / 2)\left[a^{-1} \partial_{t} u(t / 2)\right], \\
& J_{3}(t)=-\int_{0}^{\frac{t}{2}} L T(t-s)\left[a^{-1} \partial_{t} u(s)\right] d s .
\end{aligned}
$$


Hence, it suffices to estimate $J_{1}, J_{2}$ and $J_{3}$ term by term. In what follows, we shall frequently apply Theorem 5.11 with $k=1$. In the rest of the proof, we divide the proof into two cases $\lambda \in\left[\frac{2 \alpha}{2-\alpha}, \frac{N-\alpha}{2-\alpha}\right)$ and $\lambda \in\left(0, \frac{2 \alpha}{2-\alpha}\right)$.

(The case $\lambda \in\left[\frac{2 \alpha}{2-\alpha}, \frac{N-\alpha}{2-\alpha}\right)$ ) By the Schwarz inequality, Lemma 4.1 and Theorem 5.11 with the bound of $\int_{0}^{t}\left(t_{0}+s\right)^{3} \int_{\Omega} a(x)\left|\partial_{t}^{2} u(x, s)\right|^{2} \Psi\left(x, s ; t_{0}\right)^{\lambda} d x d s$, we deduce

$$
\begin{aligned}
\left\|J_{1}(t)\right\|_{L_{d \mu}^{2}}^{2} & \leq \frac{t}{2} \int_{t / 2}^{t}\left\|a^{-1}\left[\sqrt{a} \partial_{t}^{2} u(s)\right]\right\|_{L^{2}}^{2} d s \\
& \leq C t \int_{t / 2}^{t}\left\|\Psi^{\frac{\alpha}{2-\alpha}}\left[\sqrt{a} \partial_{t}^{2} u(s)\right]\right\|_{L^{2}}^{2} d s \\
& \leq C t \int_{t / 2}^{t}\left(t_{0}+s\right)^{-3-\left(\lambda-\frac{2 \alpha}{2-\alpha}\right)}\left[\left(t_{0}+s\right)^{3} \int_{\Omega} a(x)\left|\partial_{t}^{2} u(x, s)\right|^{2} \Psi\left(x, s ; t_{0}\right)^{\lambda} d x\right] d s \\
& \leq C\left(t_{0}+t\right)^{-\lambda-\frac{4(1-\alpha)}{2-\alpha}} I_{1},
\end{aligned}
$$

and hence,

$$
\left\|J_{1}(t)\right\|_{L_{d \mu}^{2}} \leq C\left(t_{0}+t\right)^{-\frac{\lambda}{2}-\frac{2(1-\alpha)}{2-\alpha}} \sqrt{I_{1}} .
$$

Applying Lemma 4.1 and Theorem 5.11 with the bound of $\left(t_{0}+t\right)^{2} \int_{\Omega} a(x)\left|\partial_{t} u(x, t)\right|^{2} \Psi\left(x, t ; t_{0}\right)^{\lambda} d x$, we have

$$
\begin{aligned}
\left\|J_{2}(t)\right\|_{L_{d \mu}^{2}} & \leq\left\|a^{-1}\left[\partial_{t} u(t / 2)\right]\right\|_{L^{2}} \\
& \leq C\left\|\Psi^{\frac{\alpha}{2-\alpha}}\left[\sqrt{a} \partial_{t} u(t / 2)\right]\right\|_{L^{2}} \\
& \leq C\left(t_{0}+t / 2\right)^{-\frac{1}{2}\left(\lambda-\frac{2 \alpha}{2-\alpha}\right)-1}\left[\left(t_{0}+t / 2\right)^{2} \int_{\Omega} a(x)\left|\partial_{t} u(x, t / 2)\right|^{2} \Psi\left(x, t / 2 ; t_{0}\right)^{\lambda} d x\right]^{\frac{1}{2}} \\
& \leq C\left(t_{0}+t\right)^{-\frac{\lambda}{2}-\frac{2(1-\alpha)}{2-\alpha}} \sqrt{I_{1}} .
\end{aligned}
$$

By Proposition 4.2 with $\sigma=\frac{\lambda}{2(2-\alpha)}-\alpha$, the Schwarz inequality and Theorem 5.5, we also estimate

$$
\begin{aligned}
& \left\|J_{3}(t)\right\|_{L_{d \mu}^{2}} \\
& \leq \int_{0}^{t / 2}\left\|L T\left(\frac{t-s}{2}\right) T\left(\frac{t-2 s}{4}\right) T\left(\frac{t}{4}\right)\left[a^{-1} \partial_{t} u(s)\right]\right\|_{L_{d \mu}^{2}} d s \\
& \leq \int_{0}^{t / 2}(t-s)^{-1}\left\|T\left(\frac{t}{4}\right)\left[a^{-1} \partial_{t} u(s)\right]\right\|_{L_{d \mu}^{2}} d s \\
& \leq C \int_{0}^{t / 2}(t-s)^{-1}\left(t_{0}+\frac{t}{4}\right)^{-\left(\frac{\lambda}{2}-\frac{\alpha}{2-\alpha}\right)}\left\|\langle x\rangle^{\frac{(2-\alpha) \lambda}{2}}\left[\sqrt{a} \partial_{t} u(s)\right]\right\|_{L^{2}} d s \\
& \leq C\left(t_{0}+t\right)^{-\left(\frac{\lambda}{2}-\frac{\alpha}{2-\alpha}\right)-1}\left(\int_{0}^{t / 2}\left(t_{0}+s\right)^{-1} d s\right)^{\frac{1}{2}}\left[\int_{0}^{t / 2}\left(t_{0}+s\right) \int_{\Omega} a(x)\left|\partial_{t} u(x, s)\right|^{2} \Psi\left(x, s ; t_{0}\right)^{\lambda} d x d s\right]^{\frac{1}{2}} \\
& \leq C\left(t_{0}+t\right)^{-\frac{\lambda}{2}-\frac{2(1-\alpha)}{(2-\alpha)}} \sqrt{\log \left(t_{0}+t\right)} \sqrt{I_{1}} .
\end{aligned}
$$


(The case $\left.\lambda \in\left(0, \frac{2 \alpha}{2-\alpha}\right)\right)$ In this case we shall use the interpolation estimates

$$
\begin{array}{r}
\left(t_{0}+t\right)^{2(1-\theta)} \int_{\Omega} a(x)\left|\partial_{t} u(x, t)\right|^{2} \Psi\left(x, t ; t_{0}\right)^{\lambda+\frac{2 \alpha}{2-\alpha} \theta} d x \leq C I_{1}, \\
\int_{0}^{t}\left(t_{0}+s\right)^{3(1-\theta)} \int_{\Omega} a(x)\left|\partial_{t}^{2} u(x, s)\right|^{2} \Psi\left(x, s ; t_{0}\right)^{\lambda+\frac{3 \alpha}{2-\alpha} \theta} d x d s \leq C I_{1}
\end{array}
$$

for $\theta \in[0,1]$, which follow from Theorem 5.11 with $k=1$ and the Hölder inequality.

For $J_{1}(t)$, applying (5.18) with $\theta=\frac{2}{3}-\frac{2-\alpha}{3 \alpha} \lambda$, we compute

$$
\begin{aligned}
\left\|J_{1}(t)\right\|_{L_{d \mu}^{2}}^{2} & \leq \frac{t}{2} \int_{t / 2}^{t}\left\|a^{-1}\left[\sqrt{a} \partial_{t}^{2} u\right]\right\|_{L^{2}}^{2} d s \\
& \leq C t \int_{t / 2}^{t}\left\|\Psi^{\frac{\alpha}{2-\alpha}}\left[\sqrt{a} \partial_{t}^{2} u\right]\right\|_{L^{2}}^{2} d s \\
& \leq C t \int_{t / 2}^{t} \int_{\Omega} a(x)\left|\partial_{t}^{2} u(x, s)\right|^{2} \Psi\left(x, s ; t_{0}\right)^{\lambda+\left(\frac{2 \alpha}{2-\alpha}-\lambda\right)} d x d s \\
& \leq C t \int_{t / 2}^{t}\left(t_{0}+s\right)^{-3(1-\theta)} \cdot\left(t_{0}+s\right)^{3(1-\theta)} \int_{\Omega} a(x)\left|\partial_{t}^{2} u(x, s)\right|^{2} \Psi\left(x, s ; t_{0}\right)^{\lambda+\frac{3 \alpha}{2-\alpha} \theta} d x d s \\
& \leq C t\left(t_{0}+t / 2\right)^{-3(1-\theta)} I_{1} \\
& \leq C\left(t_{0}+t\right)^{-\frac{2-\alpha}{\alpha} \lambda} I_{1},
\end{aligned}
$$

and hence,

$$
\left\|J_{1}(t)\right\|_{L_{d \mu}^{2}} \leq C\left(t_{0}+t\right)^{-\frac{2-\alpha}{2 \alpha} \lambda} \sqrt{I_{1}} .
$$

For $J_{2}(t)$, we have

$$
\begin{aligned}
\left\|J_{2}(t)\right\|_{L_{d \mu}^{2}} & \leq\left\|a^{-1}\left[\sqrt{a} \partial_{t} u(t / 2)\right]\right\|_{L^{2}} \\
& \leq C\left\|\Psi \Psi^{\frac{\alpha}{2-\alpha}}\left[\sqrt{a} \partial_{t} u(t / 2)\right]\right\|_{L^{2}} \\
& \leq C\left[\int_{\Omega} a(x)\left|\partial_{t} u(x, t / 2)\right|^{2} \Psi\left(x, t / 2 ; t_{0}\right)^{\lambda+\left(\frac{2 \alpha}{2-\alpha}-\lambda\right)} d x\right]^{\frac{1}{2}} \\
& \leq C\left(t_{0}+t / 2\right)^{-(1-\theta)}\left[\left(t_{0}+t / 2\right)^{2(1-\theta)} \int_{\Omega} a(x)\left|\partial_{t} u(x, t / 2)\right|^{2} \Psi\left(x, t / 2 ; t_{0}\right)^{\lambda+\frac{2 \alpha}{2-\alpha} \theta} d x\right]^{\frac{1}{2}} \\
& \leq C\left(t_{0}+t\right)^{-\frac{2-\alpha}{2 \alpha} \lambda} \sqrt{I_{1}}
\end{aligned}
$$


where we used (5.17) with $\theta=1-\frac{2-\alpha}{2 \alpha} \lambda$. Finally, for $J_{3}(t)$, we estimate

$$
\begin{aligned}
\left\|J_{3}(t)\right\|_{L_{d \mu}^{2}} & \leq \int_{0}^{t / 2}\left\|L T\left(\frac{t-s}{2}\right) T\left(\frac{t-2 s}{4}\right) T\left(\frac{t}{4}\right)\left[a^{-1} \partial_{t} u(s)\right]\right\|_{L_{d \mu}^{2}} d s \\
& \leq \int_{0}^{t / 2}(t-s)^{-1}\left\|a^{-1} \partial_{t} u(s)\right\|_{L_{d \mu}^{2}} d s \\
& \leq C t^{-1} \int_{0}^{t / 2}\left\|\Psi^{\frac{\alpha}{2-\alpha}}\left[\sqrt{a} \partial_{t} u(s)\right]\right\|_{L^{2}} d s \\
& \leq C t^{-1} \int_{0}^{t / 2}\left[\int_{\Omega} a(x)\left|\partial_{t} u(x, s)\right|^{2} \Psi\left(x, s ; t_{0}\right)^{\lambda+\left(\frac{2 \alpha}{2-\alpha}-\lambda\right)} d x\right]^{\frac{1}{2}} d s \\
& \leq C t^{-1} \int_{0}^{t / 2}\left(t_{0}+s\right)^{-(1-\theta)}\left[\left(t_{0}+s\right)^{2(1-\theta)} \int_{\Omega} a(x)\left|\partial_{t} u(x, s)\right|^{2} \Psi\left(x, s ; t_{0}\right)^{\lambda+\frac{2 \alpha}{2-\alpha} \theta} d x\right]^{\frac{1}{2}} d s \\
& \leq C\left(t_{0}+t\right)^{-1-(1-\theta)+1} \sqrt{I_{1}} \\
& \leq C\left(t_{0}+t\right)^{-\frac{2-\alpha}{2 \alpha} \lambda} \sqrt{I_{1}},
\end{aligned}
$$

where $\theta=1-\frac{2-\alpha}{2 \alpha} \lambda$. Combining all the estimates, we have the desired estimate.

Proof of Theorem 1.6. For the initial data $\left(u_{0}, u_{1}\right) \in\left(H^{2}(\Omega) \cap H_{0}^{1}(\Omega)\right) \times H_{0}^{1}(\Omega)$ satisfying $E_{0}, E_{0}^{\prime}<\infty$ with $\sigma \in\left(0, \frac{N-\alpha}{2}\right)$, we take an appropriate approximation $\left\{\left(u_{0 n}, u_{1 n}\right)\right\}_{n=1}^{\infty}$ satisfying $\left\|\left(u_{0 n}, u_{1 n}\right)\right\|_{H^{2} \times H^{1}} \leq$ $C\left\|\left(u_{0}, u_{1}\right)\right\|_{H^{2} \times H^{1}}$ and the assumptions of Theorem 5.20 with $\lambda=\frac{2 \sigma}{2-\alpha}$ (see [23, p.611] for the detail). Then, applying Theorem 5.20 to $\left(u_{0 n}, u_{1 n}\right)$ and taking the limit $n \rightarrow \infty$, we have the desired conclusion.

\section{Acknowledgements}

This work was supported by JSPS KAKENHI Grant Numbers JP18K134450, JP16K17625, JP18H01132. 


\section{References}

[1] R. Beals, R. Wong, "Special functions," A graduate text. Cambridge Studies in Advanced Mathematics 126, Cambridge University Press, Cambridge, 2010.

[2] C. Cattaneo, Sur une forme de l'équation de la chaleur éliminant le paradoxe d'une propagation instantanée, C. R. Acad. Sci. 247 (1958), 431-433.

[3] R. Chill, A. Haraux, An optimal estimate for the difference of solutions of two abstract evolution equations, J. Differential Equations 193 (2003), 385-395.

[4] K.-J. Engel, R. Nagel, "One-parameter semigroups for linear evolution equations," Graduate Texts in Mathematics, 194, Springer-Verlag, New York, 2000.

[5] D. Gilbarg, N.S. Trudinger, "Elliptic partial differential equations of second order," Grundlehren der Mathematischen Wissenschaften, Vol. 224. Springer-Verlag, Berlin-New York, 1977.

[6] L. Hsiao, T. -P. Liu, Convergence to nonlinear diffusion waves for solutions of a system of hyperbolic conservation laws with damping, Comm. Math. Phys. 43 (1992), 599-605.

[7] M. Ikawa, Mixed problems for hyperbolic equations of second order, J. Math. Soc. Japan 20 (1968), 580-608.

[8] R. Ikehata, Diffusion phenomenon for linear dissipative wave equations in an exterior domain, J. Differential Equations 186 (2002), 633-651.

[9] K. Ishige, T. Kawakami, M. Sierżȩga, Supersolutions for a class of nonlinear parabolic systems, J. Differential Equations 260 (2016), 6084-6107.

[10] N. Ioku, G. Metafune, M. Sobajima, C. Spina, $L^{p}-L^{q}$ estimates for homogeneous operators, Commun. Contemp. Math. 18 (2016), no. 3, 1550037, 14 pp.

[11] G. Karch, Selfsimilar profiles in large time asymptotics of solutions to damped wave equations, Studia Math. 143 (2000), 175-197.

[12] H.A. Levine, Fujita type theorems for weakly coupled parabolic systems, Progress in partial differential equations: elliptic and parabolic problems (Pont-à-Mousson, 1991), 43-55, Pitman Res. Notes Math. Ser. 266, Longman Sci. Tech., Harlow, 1992.

[13] G. Lu, B.D. Sleeman, Subsolutions and supersolutions to systems of parabolic equations with applications to generalized Fujita-type systems, Math. Methods Appl. Sci. 17 (1994), 1005-1016.

[14] K. Nishihara, $L^{p}-L^{q}$ estimates of solutions to the damped wave equation in 3-dimensional space and their application, Math. Z. 244 (2003), 631-649.

[15] H. Nishiyama, Remarks on the asymptotic behavior of the solution to damped wave equations, J. Differential Equations 261 (2016) 3893-3940.

[16] P. Radu, G. Todorova, B. Yordanov, Higher order energy decay rates for damped wave equations with variable coefficients, Discrete Contin. Dyn. Syst. Ser. S. 2 (2009), 609-629. 
[17] P. Radu, G. Todorova, B. Yordanov, Decay estimates for wave equations with variable coefficients, Trans. Amer. Math. Soc. 362 (2010), 2279-2299.

[18] P. Radu, G. Todorova, B. Yordanov, The generalized diffusion phenomenon and applications, SIAM J. Math. Anal. 48 (2016), 174-203.

[19] P. Quittner, P. Souplet, "Superlinear parabolic problems, Blow-up, global existence and steady states," Birkhäuser Advanced Texts: Basler Lehrbücher. [Birkhäuser Advanced Texts: Basel Textbooks] Birkhäuser Verlag, Basel, 2007.

[20] M. Sobajima, Global existence of solutions to semilinear damped wave equation with slowly decaying initial data in exterior domain, arXiv:1812.10664v1.

[21] M. Sobajima, Y. Wakasugi, Diffusion phenomena for the wave equation with space-dependent damping in an exterior domain, J. Differential Equations 261 (2016), 5690-5718.

[22] M. Sobajima, Y. Wakasugi, Remarks on an elliptic problem arising in weighted energy estimates for wave equations with space-dependent damping term in an exterior domain, AIMS Mathematics, 2 (2017), 1-15.

[23] M. Sobajima, Y. Wakasugi, Diffusion phenomena for the wave equation with space-dependent damping term growing at infinity, Adv. Differential Equations 23 (2018), 581-614.

[24] M. Sobajima, Y. Wakasugi, Weighted energy estimates for wave equation with space-dependent damping term for slowly decaying initial data, Commun. Contemp. Math. 21 (2019), no. 5, $1850035,30 \mathrm{pp}$.

[25] G. Todorova, B. Yordanov, Weighted $L^{2}$-estimates for dissipative wave equations with variable coefficients, J. Differential Equations 246 (2009), 4497-4518.

[26] P. Vernotte, Les paradoxes de la théorie continue de l'équation de la chaleur, Comptes Rendus 246 (1958), 3154-3155.

[27] Y. Wakasugi, On diffusion phenomena for the linear wave equation with space-dependent damping, J. Hyp. Diff. Eq. 11 (2014), 795-819.

[28] E.B. Weissler, Single point blow-up for a semilinear initial value problem, J. Differential Equations 55 (1984), 204-224.

[29] H. Yang, A. Milani, On the diffusion phenomenon of quasilinear hyperbolic waves, Bull. Sci. Math. 124 (2000), 415-433. 